BMI-1422

\title{
NUMERICAL SOLUTION OF FUEL-ELEMENT THERMAL-STRESS PROBLEMS
}

BATTELLE MEMORIAL INSTITUTE 


\section{LEGAL NOTICE}

This report was prepared as an account of Government sponsored work. Neither the United States, nor the Commission, nor any person acting on behalf of the Commission:

A. Makes any warranty or representation, expressed or implied, with respect to the accuracy, completeness, or usefulness of the information contained in this report, or that the use of any information, apparatus, method, or process disclosed in this report may not infringe privately owned rights; or

B. Assumes any liabilities with respect to the use of, or for damages resulting from the use of any information, apparatus, method, or process disclosed in this report.

As used in the above, "person acting on behalf of the Commission" includes any employee or contractor of the Commission, or employee of such contractor, to the extent that such employee or contractor of the Commission, or employee of such contractor prepares, disseminates, or provides access to, any information pursuant to his employment or contract with the Commission, or his employment with such contractor.

Printed in USA

Price $\$ 1.50$

Available from the Office of Technical Services U. S. Department of Commerce Wa shington 25. D. C. 


\section{DISCLAIMER}

This report was prepared as an account of work sponsored by an agency of the United States Government. Neither the United States Government nor any agency Thereof, nor any of their employees, makes any warranty, express or implied, or assumes any legal liability or responsibility for the accuracy, completeness, or usefulness of any information, apparatus, product, or process disclosed, or represents that its use would not infringe privately owned rights. Reference herein to any specific commercial product, process, or service by trade name, trademark, manufacturer, or otherwise does not necessarily constitute or imply its endorsement, recommendation, or favoring by the United States Government or any agency thereof. The views and opinions of authors expressed herein do not necessarily state or reflect those of the United States Government or any agency thereof. 


\section{DISCLAIMER}

Portions of this document may be illegible in electronic image products. Images are produced from the best available original document. 
Report No. BMI-1422

UC-34 Physics and Mathematics (TID-4500, 15th Ed.)

Contract No. W-7405-eng-92

\title{
NUMERICAL SOLUTION OF FUEL-ELEMENT THERMAL-STRESS PROB LEMS
}

\author{
by
}

Robert F. Redmond

Harry Pollack

Alton E. Klickman

William S. Hogan

Harold M. Epstein

Joel W. Chastain

February 26, 1960

BATTELLE MEMORIAL INSTITUTE

$505 \mathrm{King}$ Avenue

Columbus 1, Ohio 
TABLE OF CONTENTS

$\underline{\text { Page }}$

ABSTRACT . . . . . . . . . . . . . . . . . . . . . . . . . . 1

INTRODUCTION . . . . . . . . . . . . . . . . . . . . . . . . . . . .

FORMULATION OF THE PROBLEM . . . . . . . . . . . . . . . . . . . . 2

Differential Equations for the Temperature Field . . . . . . . . . . 2

Differential Equations for the Stress Field . . . . . . . . . . . . . 3

Formulation of Equations for Numerical Solution. . . . . . . . . . 9

Construction of Mesh . . . . . . . . . . . . . . . . 10

Interpolation Formulas . . . . . . . . . . . . . . 10

Numerical Equations .. . . . . . . . . . . . . 13

ITERATIVE METHOD OF SOLUTION . . . . . . . . . . . . . . . . . . 15

Minimization Process for Iteration . . . . . . . . . . . . . . 16

The Extrapolated Liebmann Process . . . . . . . . . . . . . 18

Terminal Block Extrapolation . . . . . . . . . . . . . . . . 20

NUMERICAL RESULTS . . . . . . . . . . . . . . . . . . . . . . 23

Preliminary Studies . . . . . . . . . . . . . . . . . 23

Fuel-Element Studies . . . . . . . . . . . . . . . . 30

Single-Hole Hexagonal Element . . . . . . . . . . . 30

Seven-Hole Ilexagonal Element . . . . . . . . . . . . . . 31

Plate-Type Element . . . . . . . . . . . . . . . . . 31

DISCUSSION OF RESULTS AND CONCLUSIOINS . . . . . . . . . . . . . . . 34

REFERENCES. . . . . . . . . . . . . . . . . . . . . . 35

APPENDIX A

SETUP OF PROBLEM FOR MACHINE COMPUTATION . . . . . . . . . . . A-l 


\title{
NUMERICAL SOLUTION OF FUEL-ELEMENT THERMAL-STRESS PROB LEMS
}

\author{
Robert F. Redmond, Harry Pollack, Alton E. Klickman, \\ William S. Flogan, Harold M. Epstein, \\ and Joel W. Chastain
}

\begin{abstract}
In developing a method of numerical analysis for the solution of thermalstress problems special emphasis uas given to fuel elements with internal coolant channels. Numerical techniques for reducing the partial differential equation system to a form suitable for numerical solution and a new iterative method of solving large systems of linear algebraic equations were employed. Computer codes were devised to obtain the numerical solution of the thermal-stress problems and were used to obtain numerical results for single-hole and seven-hole hexagonal elements and plate-type elements. Comparisons were made between analytical results and numerical results for the case of the simple annulus shape.
\end{abstract}

\section{INTRODUCTION}

The performance of reactor fuel elements is affected and frequently limited by the occurrence of thermal stresses. Hence, a design problem constantly encountered is the prediction of the thermal stresses which will occur in a given reactor fuel element. The problem of analyzing for the thermal stresses is usually complicated by the geometry of the fuel elements. The fuel elements may have holes and will probably require a twodimensional description.

This report describes a general method of numerical analysis which can be applied to a wide range of two-dimensional thermal-stress problems. These numerical methods have been coded for the IBM-704 computer and have been used to analyze a number of fuel-element geometries.

The procedure followed is to develop the appropriate partial differential equations which describe the problem, to approximate the differential equations by difference equations, and then to solve the resulting difference equations. The major difficulty normally encountered in this procedure is the solution of the difference equations. There may be as many as 500 to 1000 linear algebraic equations to solve simultaneously, and this is a formidable task even for the IBM-704. The method of solution adopted in this work was an iterative procedure. This method has advantages and disadvantages which will be discussed later.

The remaining parts of this report give the details of the problem formulation, the iterative method of solution, and the numerical results. Finally, the methods and results are evaluated and discussed. 


\section{FORMULATION OF THE PROBLEM}

The problem considered is that of determining the plane-strain thermal-stress distributions in cylindrical bodies which may have irregular boundaries and which may contain cylindrical holes. It is assumed that all physical properties are constant in the problem and that the material is isotropic and homogeneous. The body is assumed to have uniform internal heat generation and to be cooled by heat transfer to a coolant. The heat-transfer coefficient is assumed to be constant.

The assumptions regarding the heat generation and heat transfer could easily be modified if more detailed information were available. The results also will apply to plane-stress problems with minor changes in the coefficients.

Throughout this report the body under study will be oriented in such a way that the $z$-axis is parallel to the cylindrical body and the cross section of the body will be depicted in the $x y$-plane. All quantities will be assumed to be independent of the $z$ coordinate.

\section{Differential Equations for the Temperature Field}

The analysis of the thermal-stress problem can be broken down into two parts. First, the temperature distribution must usually be determined; and, second, the resulting thermal stresses must be determined. Consider first the two-dimensional temperature problem in the $x y$ plane. The equations to be satisfied by the temperature are as follows:

$$
\begin{gathered}
\frac{\partial^{2} \mathrm{~T}(\mathrm{x}, \mathrm{y})}{\partial \mathrm{x}^{2}}+\frac{\partial^{2} \mathrm{~T}(\mathrm{x}, \mathrm{y})}{\partial \mathrm{y}^{2}}=\nabla^{2} \mathrm{~T}(\mathrm{x}, \mathrm{y})=-\frac{\mathrm{Q}}{\mathrm{k}}, \\
\frac{\partial \mathrm{T}\left(\mathrm{x}_{\mathrm{s}}, \mathrm{y}_{\mathrm{s}}\right)}{\partial \mathrm{n}}=0 \text {, at insulated or symmetry surfaces, } \\
\frac{\partial \mathrm{T}\left(\mathrm{x}_{\mathrm{s}}, \mathrm{y}_{\mathrm{s}}\right)}{\partial \mathrm{n}}=-\frac{\mathrm{h}}{\mathrm{k}} \mathrm{T}\left(\mathrm{x}_{\mathrm{s}}, \mathrm{y}_{\mathrm{s}}\right) \text {, at heat-transfer surfaces, }
\end{gathered}
$$

where

$$
\begin{aligned}
T(x, y)= & \text { temperature above the mean coolant temperature at the point } \\
& (\mathrm{x}, \mathrm{y}) \text { in the body } \\
Q= & \text { volumetric heat source } \\
\mathrm{k}= & \text { thermal conductivity } \\
\mathrm{h}= & \text { heat-transfer coefficient } \\
\left(\mathrm{x}_{\mathrm{S}}, \mathrm{y}_{\mathrm{S}}\right)= & \text { coordinates of a point on the surface }
\end{aligned}
$$




$$
\frac{\partial}{\partial n}=\text { normal derivative in the direction outward from the surface. }
$$

These equations are more convenient for numerical work when they are expressed in dimensionless form as follows:

$$
\begin{gathered}
\frac{\partial^{2} \overline{\mathrm{T}}(\overline{\mathrm{x}}, \overline{\mathrm{y}})}{\partial \overline{\mathrm{x}}^{2}}+\frac{\partial^{2} \overline{\mathrm{T}}(\overline{\mathrm{x}}, \overline{\mathrm{y}})}{\partial \overline{\mathrm{y}}^{2}}=\nabla^{2} \overline{\mathrm{T}}(\overline{\mathrm{x}}, \overline{\mathrm{y}})=-1, \\
\frac{\partial \overline{\mathrm{T}}\left(\overline{\mathrm{x}}_{\mathrm{s}}, \overline{\mathrm{y}}_{\mathrm{s}}\right)}{\partial \overline{\mathrm{n}}^{2}}=0, \text { at insulated or symmetry surfaces }, \\
\frac{\partial \overline{\mathrm{T}}\left(\overline{\mathrm{x}}_{\mathrm{S}}, \overline{\mathrm{y}}_{\mathrm{s}}\right)}{\partial \overline{\mathrm{n}}^{\mathrm{n}}}=-\overline{\beta \mathrm{T}}\left(\overline{\mathrm{x}}_{\mathrm{S}}, \overline{\mathrm{y}}_{\mathrm{s}}\right), \text { at heat-transfer surfaces },
\end{gathered}
$$

where

$$
\begin{aligned}
\bar{x} & =\frac{x}{c}, \bar{y}=\frac{y}{c}, \bar{n}=\frac{n}{c} \\
c & =\text { characteristic length } \\
\bar{T}(\bar{x}, \bar{y}) & =\frac{T(x, y)}{\frac{Q c^{2}}{k}} \\
\beta & =\frac{h c}{k} .
\end{aligned}
$$

Equations (lb) are the equations which will be used for the temperature field throughout the remainder of this report.

\section{Differential Equations for the Stress Field}

The equations for the stress field in the case of plane strain in the absence of body forces are as follows (1):

$$
\begin{gathered}
\frac{\partial \sigma_{x}}{\partial x}+\frac{\partial \tau_{x y}}{\partial y}=0 \\
\frac{\partial \tau_{x y}}{\partial x}+\frac{\partial \sigma_{y}}{\partial y}=0 \\
\epsilon_{x}=\frac{1}{E}\left[(1+\nu) \sigma_{x}-\nu \Theta\right]+\alpha T, \\
\epsilon_{y}=\frac{1}{E}\left[(1+\nu) \sigma_{y}-\nu \Theta\right]+\alpha T, \\
\epsilon_{z}=\frac{1}{E}\left[(1+\nu) \sigma_{z}-\nu \Theta\right]+\alpha T=\text { constant } \\
\gamma_{x y}=\frac{2(1+\nu)}{E} \tau_{x y},
\end{gathered}
$$


where

$$
\begin{aligned}
\sigma_{\mathrm{x}} & =\text { normal stress component on surface normal to the } \mathrm{x} \text {-axis } \\
\sigma_{\mathrm{y}} & =\text { normal stress component on surface normal to the y-axis } \\
\sigma_{\mathrm{z}} & =\text { normal stress component on surface normal to the z-axis } \\
\tau_{\mathrm{xy}} & =\text { tangential stress in } \mathrm{y} \text {-direction on surface normal to } \mathrm{x} \text {-axis } \\
\epsilon_{\mathrm{x}} & =\text { strain in } \mathrm{x} \text {-direction } \\
\epsilon_{\mathrm{y}} & =\text { strain in } \mathrm{y} \text {-direction } \\
\epsilon_{\mathrm{z}} & =\text { strain in } \mathrm{z} \text {-direction } \\
\gamma_{\mathrm{xy}} & =\text { shear strain in } \mathrm{xy}-\text { plane } \\
\Theta & =\sigma_{\mathrm{x}}+\sigma_{\mathrm{y}}+\sigma_{\mathrm{z}} \\
\nu & =\text { Poisson's ratio } \\
\mathrm{E} & =\text { Young's modulus } \\
\alpha & =\text { linear coefficient of thermal expansion } \\
\mathrm{T} & =\text { temperature. }
\end{aligned}
$$

Equations (2a) represent a force balance on a small-volume element of the body, whereas Equations (2b) are the relationships between stress and strain. In addition to these equations, the strains must satisfy certain compatibility conditions in order to make the strains consistent with the displacements. Further, the stress-strain fields must be such that the displacements and rotations are continuous where the body is continuous. Finally, the stresses must be consistent with the externally applied forces at the surface of the body.

In the present situation the only compatibility equation which is not satisfied trivially is

$$
\frac{\partial^{2} \epsilon_{x}}{\partial y^{2}}+\frac{\partial^{2} \epsilon_{y}}{\partial x^{2}}=\frac{\partial^{2} \gamma_{x y}}{\partial x \partial y}
$$

Using Equations (2b) in Equation (3) leads to the following equation involving the stresses

$$
\frac{\partial^{2} \sigma_{y}}{\partial x^{2}}-\frac{2 \partial^{2} \tau_{x y}}{\partial x \partial y}+\frac{\partial^{2} \sigma_{x}}{\partial y^{2}}-\nu \nabla^{2}\left(\sigma_{x}+\sigma_{y}\right)=-\alpha E \nabla^{2} T .
$$

Forgetting for the moment the additional equations which may be needed, consider Equations (2a) and (4) which the stresses must satisfy. As a matter of convenience a stress function $\phi(x, y)$ is usually defined with the following properties: 


$$
\sigma_{x}=\frac{\partial^{2} \phi}{\partial y^{2}} \quad, \quad \sigma_{y}=\frac{\partial^{2} \phi}{\partial x^{2}} \quad, \quad \tau_{x y}=-\frac{\partial^{2} \phi}{\partial x \partial y}
$$

First, it will be noted that Equations (2a) are satisfied trivially. On the other hand, Equation (4) will be satisfied if $\phi$ is a solution of the equation

$$
\nabla^{4} \phi(\mathrm{x}, \mathrm{y})=\nabla^{2} \nabla^{2} \phi(\mathrm{x}, \mathrm{y})=\frac{-\alpha \mathrm{E}}{1-\nu} \nabla^{2} \mathrm{~T}
$$

which, if $\nabla^{2} \mathrm{~T}=-\frac{\mathrm{Q}}{\mathrm{k}}$, becomes

$$
\nabla^{4} \phi(\mathrm{x}, \mathrm{y})=\left(\frac{\alpha \mathrm{E}}{1-\nu}\right)\left(\frac{\mathrm{Q}}{\mathrm{k}}\right)
$$

Therefore, a solution for $\phi$ which satisfies Equation (6b) and the additional conditions to be required will determine, by Equation (5), $\sigma_{x}, \sigma_{y}$, and $\tau_{x y}$. The remaining stress $\sigma_{z}$ does not appear in Equations (2a) and (4) but is determined as follows. From the $\epsilon_{\mathrm{z}}$ equation of Equations (2b), $\sigma_{\mathrm{Z}}$ is expressed in terms of the other stresses, the temperature, and $\epsilon_{\mathrm{z}}$ (a constant):

$$
\begin{gathered}
\sigma_{z}=E\left(\epsilon_{z}-\alpha T\right)+\nu\left(\sigma_{x}+\sigma_{y}\right), \\
\sigma_{z}=E\left(\epsilon_{z}-\alpha T\right)+\nu \nabla^{2} \phi .
\end{gathered}
$$

It is then required that the $\sigma_{z}$ component be consistent with the axial forces acting on the body. If the body is unrestrained at the ends, as is the case here, then

$$
\iint \sigma_{z} d x d y=\iint\left\{E\left(\epsilon_{z}-\alpha T\right)+\nu \nabla^{2} \phi\right\} d x d y=0
$$

where the integrals extend over the cross-sectional area of the body. From Equation (8) $\epsilon_{\mathrm{z}}$ can be determined and then $\sigma_{\mathrm{z}}$ can be evaluated from Equation (7).

In many cases it is easy to show by Green's theorem that $\int \nabla^{2} \phi \mathrm{dxdy}=0$ and, hence, $\epsilon_{\mathrm{z}}=\alpha \mathrm{T}_{\mathrm{av}}$, where $\mathrm{T}_{\mathrm{av}}$ is the average temperature of the body. In this case $\sigma_{\mathrm{z}}$ is given simply by

$$
\sigma_{z}=\alpha E\left(T_{a V}-T\right)+\nu \nabla^{2} \phi
$$

In addition to the preceding equations certain boundary conditions and continuity conditions must be satisfied. At the free boundaries of the body the stresses must be consistent with the applied forces. Hence, at the free boundaries,

$$
\begin{aligned}
& \mathrm{n}_{\mathrm{x}} \sigma_{\mathrm{x}}+\mathrm{n}_{\mathrm{y}} \tau_{\mathrm{xy}}=\mathrm{F}_{\mathrm{x}} \\
& \mathrm{n}_{\mathrm{x}} \tau_{\mathrm{xy}}+\mathrm{n}_{\mathrm{y}} \sigma_{\mathrm{y}}=\mathrm{F}_{\mathrm{y}}
\end{aligned}
$$

where $n_{X}$ and $n_{y}$ are the direction cosines of the outward normal vector, and $F_{x}$ and $F_{y}$ are the components of the externally applied surface force. In terms of the stress function, 


$$
\begin{gathered}
n_{x} \frac{\partial^{2} \phi}{\partial y^{2}}-n_{y} \frac{\partial^{2} \phi}{\partial x \partial y}=\frac{d}{d s}\left(\frac{\partial \phi}{\partial x}\right)=F_{x} \\
-n_{x} \frac{\partial^{2} \phi}{\partial x \partial y}+n_{y} \frac{\partial^{2} \phi}{\partial x^{2}}=-\frac{d}{d s}\left(\frac{\partial \phi}{\partial y}\right)=F_{y}
\end{gathered}
$$

where $\frac{d}{d s}$ represents the derivative in the direction of the tangent vector of the boundary curve. For free boundaries $F_{x}=F_{y}=0$ and, hence,

$$
\begin{aligned}
& \frac{\partial \phi}{\partial x}=\text { constant }, \\
& \frac{\partial \phi}{\partial y}=\text { constant },
\end{aligned}
$$

on each free boundary separately. In many cases considered, one or both constants in Equation (9c) are necessarily zero from a consideration of the symmetry planes which intersect the free boundaries.

In addition to the free boundaries just considered, symmetry boundaries may sometimes be introduced to simplify the problem. In this case the stress function is required to be symmetrical with respect to the symmetry surface. At a symmetry surface the following conditions are equivalent:

$$
\left\{\begin{array} { c } 
{ \frac { \partial \phi } { \partial \mathrm { n } } = 0 , } \\
{ \frac { \partial \nabla ^ { 2 } \phi } { \partial \mathrm { n } } = 0 , }
\end{array} \text { or } \left\{\begin{array}{c}
\frac{\partial \phi}{\partial \mathrm{n}}=0, \\
\frac{\partial^{3} \phi}{\partial \mathrm{n}^{3}}=0,
\end{array}\right.\right.
$$

where $\frac{\partial}{\partial n}$ represents a normal derivative at the symmetry surface.

For simply connected bodies (no holes) Equations (6a), (9b), and (10) would suffice to determine the stress function for the resulting displacements and rotations would satisfy the continuity requirement. However, for multiply connected bodies (holes), additional conditions must be placed upon the stress function to assure continuity of the displacements and the rotations.

The following treatment follows that of Southwell(2). The strain components in terms of the displacements are

$$
\epsilon_{\mathrm{x}}=\frac{\partial \mathrm{u}}{\partial \mathrm{x}}, \quad \epsilon_{\mathrm{y}}=\frac{\partial \mathrm{v}}{\partial \mathrm{y}}, \quad \gamma_{\mathrm{xy}}=\frac{\partial \mathrm{u}}{\partial \mathrm{y}}+\frac{\partial \mathrm{v}}{\partial \mathrm{x}},
$$

where

$$
\begin{aligned}
& \mathrm{u}=\text { displacement component in } \mathrm{x} \text {-direction } \\
& \mathrm{v}=\text { displacement component in } \mathrm{y} \text {-direction. }
\end{aligned}
$$


The only rotational component of interest here is the $z$-component, $w_{z}$, given by

$$
\mathrm{w}_{z}=\frac{1}{2}\left(\frac{\partial v}{\partial x}-\frac{\partial u}{\partial y}\right)
$$

Hence,

$$
\begin{aligned}
& \frac{\partial v}{\partial x}=w_{z}+\frac{1}{2} \gamma_{x y} \\
& \frac{\partial u}{\partial y}=-w_{z}+\frac{1}{2} \gamma_{x y}
\end{aligned}
$$

and

$$
\begin{aligned}
& \frac{\partial}{\partial y}\left(w_{z}+\frac{1}{2} \gamma_{x y}\right)=\frac{\partial^{2} v}{\partial x \partial y}=\frac{\partial \epsilon_{y}}{\partial x}, \\
& \frac{\partial}{\partial x}\left(-w_{z}+\frac{1}{2} \gamma_{x y}\right)=\frac{\partial^{2} u}{\partial x \partial y}=\frac{\partial \epsilon_{x}}{\partial y},
\end{aligned}
$$

and

$$
\begin{gathered}
\frac{\partial w_{z}}{\partial y}=\frac{\partial \epsilon_{y}}{\partial x}-\frac{1}{2} \frac{\partial \gamma_{x y}}{\partial y}, \\
\frac{\partial w_{z}}{\partial x}=-\frac{\partial \epsilon_{x}}{\partial y}+\frac{1}{2} \frac{\partial \gamma_{x y}}{\partial x} .
\end{gathered}
$$

Evaluating $\frac{\partial^{2} w_{z}}{\partial x \partial y}$ with each of the preceding expressions and equating these expressions leads to the compatibility relation already considered, Equation (3). In addition, however, if $\mathrm{w}_{\mathrm{z}}$ is continuous then

$$
\oint \frac{\partial w_{z}}{\partial s} d s=0
$$

where the path of integration is an arbitrary closed curve which does not cross the boundaries of the body but may enclose holes. In terms of the strains

$$
\begin{aligned}
\oint \frac{\partial w_{z}}{\partial s} d s & =\oint\left\{-n_{y} \frac{\partial w_{z}}{\partial x}+n_{x} \frac{\partial w_{z}}{\partial y}\right\} d s \\
& =\oint\left\{n_{x}\left(\frac{\partial \epsilon_{y}}{\partial x}-\frac{1}{2} \frac{\partial \gamma_{x y}}{\partial y}\right)+n_{y}\left(\frac{\partial \epsilon_{x}}{\partial y}-\frac{1}{2} \frac{\partial \gamma_{x y}}{\partial x}\right)\right\} d s=0 .
\end{aligned}
$$

In terms of the stress function [express $\epsilon_{\mathrm{x}}$ and $\epsilon_{\mathrm{y}}$ in terms of $\sigma_{\mathrm{x}}$ and $\sigma_{\mathrm{y}}$ by eliminating $\sigma_{z}$ by means of Equation (7) ]:

$$
\oint \frac{\partial}{\partial n}\left\{\nabla 2 \phi+\frac{\alpha E T}{1-\nu}\right\} d s=0
$$


Hence, Equation (11) must be satisfied if the rotation is to be continuous. It may be noted by applying Green's theorem to Equation (11) that

$$
\begin{aligned}
& \oint_{s} \frac{\partial}{\partial n}\left\{\nabla^{2} \phi+\frac{\alpha E T}{1-\nu}\right\} d s=\iint \nabla^{2}\left\{\nabla^{2} \phi+\frac{\alpha E T}{1-\nu}\right\} d x d y+ \\
& -\sum_{i} \oint_{s_{i}} \frac{\partial}{\partial n}\left\{\nabla^{2} \phi+\frac{\alpha E T}{1-\nu}\right\} d s=0,
\end{aligned}
$$

where in the last summation of integrals the integrations are over the paths which define the holes enclosed by the closed path $s$, and the surface integral extends over the region of the body enclosed by the path s. From Equation (6a) the surface integral vanishes. If no holes are present Equation (11) will be satisfied trivially for any path of integration in the body which, however, may coincide with the external boundary of the body as a limiting case. If holes are present then a path of integration can be defined which includes only one hole, and then the preceding arguments show that Equation (11) must be satisfied for this path of integration or for any path which includes this hole; for example, a path defined over the hole boundary. Therefore, if holes are present the continuity of $\mathrm{w}_{\mathbf{z}}$ is assured if Equation (11) is satisfied for each boundary curve. Equation (11) then is another condition imposed on the stress function which must be satisfied at each hole boundary if holes are present.

In a similar fashion the continuity of $\mathrm{u}$ and $\mathrm{v}$ can be established by requiring that

$$
\oint \frac{\partial u}{\partial s} d s=0
$$

and

$$
\oint \frac{\partial v}{\partial s} d s=0
$$

These requirements result in the following equations in terms of the stress function:

$$
\begin{aligned}
& \oint\left[\mathrm{y} \frac{\partial}{\partial \mathrm{n}}-\mathrm{n}_{\mathrm{y}}\right]\left[\nabla^{2} \phi+\frac{\alpha \mathrm{ET}}{1-\nu}\right] \mathrm{ds}=0, \\
& \oint\left[\mathrm{x} \frac{\partial}{\partial \mathrm{n}}-\mathrm{n}_{\mathrm{x}}\right]\left[\nabla^{2} \phi+\frac{\alpha \mathrm{ET}}{1-\nu}\right] \mathrm{ds}=0 .
\end{aligned}
$$

Again by an application of Green's theorem it can be seen that these equations are satisfied trivially if no holes are present. If holes are present Equations (13) and (14) must be satisfied for each hole boundary. Hence, Equations (12), (13), and (14) are to be satisfied for each hole boundary in order to insure the continuity of the displacement and the rotation components.

In addition to the continuity equations just described, the presence of holes can also introduce the problem of cyclic constants. (2) However, if, as in the present case, the externally applied forces at the hole boundaries are zero then there is no problem. Therefore, the problem of cyclic constants will not be considered further in this report. 
Finally, it must be noted that, in general, there will be solutions of the homogeneous equation $\nabla^{4} \phi=0$ which will also satisfy the boundary conditions and the continuity equations; e. g., $\phi=$ constant. In such instances the resulting arbitrary constants must be specified before the numerical work can proceed. That is, the stress function must have a unique solution if it is to be determined by a numerical procedure.

As in the case of the temperature problem dimensionless forms are more convenient. Hence, Equations (6b), (8), (10), (11), etc. become

$$
\begin{aligned}
& \bar{\nabla} 4 \bar{\phi}=1 \\
& \iint\left\{\left(\frac{\epsilon_{\mathrm{z}}}{\alpha \frac{\mathrm{Qc}^{2}}{\mathrm{k}}}-\overline{\mathrm{T}}\right)+\frac{\nu}{1-\nu} \bar{\nabla} 2 \bar{\phi}\right\} \mathrm{d} \overline{\mathrm{x}} \mathrm{d} \overline{\mathrm{y}}=0, \\
& \frac{\mathrm{d}}{\mathrm{d} \overline{\mathrm{s}}}\left(\frac{\partial \bar{\phi}}{\partial \overline{\mathrm{x}}}\right)=0, \frac{\mathrm{d}}{\mathrm{d} \overline{\mathrm{s}}}\left(\frac{\partial \bar{\phi}}{\partial \overline{\mathrm{y}}}\right)=0, \\
& \frac{\partial \bar{\phi}}{\partial \bar{n}}=0, \quad \frac{\partial \bar{\nabla}^{2} \bar{\phi}}{\partial \bar{n}}=0 \\
& \oint \frac{\partial}{\partial \bar{n}}\left\{\bar{\nabla}^{2} \bar{\phi}+\overline{\mathrm{T}}\right\} \mathrm{d} \overline{\mathrm{s}}=0 \text {, etc. , }
\end{aligned}
$$

where

$$
\bar{\phi}=\frac{\phi}{\frac{\alpha E}{1-\nu} \frac{\mathrm{Q}}{\mathrm{k}} c^{4}},
$$

and the other terminology is as before.

The dimensionless form of the equations will be used throughout the remainder of this report. However, for simplicity the bar designation will be dropped with the understanding that all equations which follow are in dimensionless form.

\section{Formulation of Equations for Numerical Solution}

The procedure used in arriving at a numerical solution of the thermal-stress problem is as follows:

(1) Construct a square mesh which is superposed over the twodimensional geometry of the body.

(2) Specify appropriate interpolation formulas for representing a function of $x$ and $y$ in terms of the point values at the mesh points.

(3) Perform the required differential and integral operations on the interpolation formulas to obtain suitable equations involving the point values of the function at the mesh points. 
(4) Solve the resulting equations to obtain the point values of the function at the mesh points and if necessary use the point values for further operations.

These steps of the procedure will now be described in turn. In order to make the procedure clear a specific example will be used. As an example a 15-deg slice of an annular cylinder will be considered. In this example it will be assumed that heat is generated uniformly within the annulus and is removed uniformly at the inner radius by heat transfer to a coolant.

\section{Construction of the Mesh}

A geometrical square mesh of unit length is superposed over the two-dimensional geometry of the body as indicated in Figure 1. Two types of physical mesh points are now defined. Interior mesh points are defined to be those on or inside the boundaries of the body. These are indicated in Figure 1 by solid dots. Exterior mesh points are defined to be those points outside the boundaries of the body which are required to complete the "biharmonic array" for each interior mesh point considered as the center of the biharmonic array. The biharmonic array is defined by the geometrical array shown in Figure 2a. The $(0,0)$ point is the center of the array. In Figure 1 the exterior points are indicated by circles at the appropriate mesh points. It will be noted that each interior point in Figure 1 is the center of a complete biharmonic array. The "Laplacian array" as shown in Figure 2b would suffice for the temperature-field problems; however, for the purpose of constructing the mesh only once for both the temperature and stress problems, the method described will be used throughout. For the temperature problems the exterior points not needed can be eliminated in the problem solution.

The use of exterior points may appear strange since function values at these points have no physical significance. However, they do have mathematical significance since they correspond to an analytic continuation of the function beyond the boundaries. For example, the analytical formula for the temperature field has physical significance only when it is evaluated for points inside the body but it may nevertheless be used to evaluate function values outside the body. The reason for using exterior points in this way is that the boundary equations can be written more accurately for numerical computation. This is because central difference interpolation formulas can be used even at the boundaries, whereas without the exterior mesh points one-sided interpolation formulas would be required, and these are less satisfactory.

Interpolation Formulas

The two-dimensional interpolation formulas given here are obtained by a double application of Stirling's(3) interpolation formula and truncating the result. For the temperature function the five-point Laplacian array is used which is associated with the following interpolation formula

$$
\begin{aligned}
T(r, s)= & T(0,0)+\frac{r}{2}[T(1,0)-T(-1,0)]+\frac{s}{2}[T(0,1)-T(0,-1)] \\
& +\frac{r^{2}}{2}[T(1,0)+T(-1,0)-2 T(0,0)] \\
& +\frac{s^{2}}{2}[T(0,1)+T(0,-1)-2 T(0,0)] .
\end{aligned}
$$




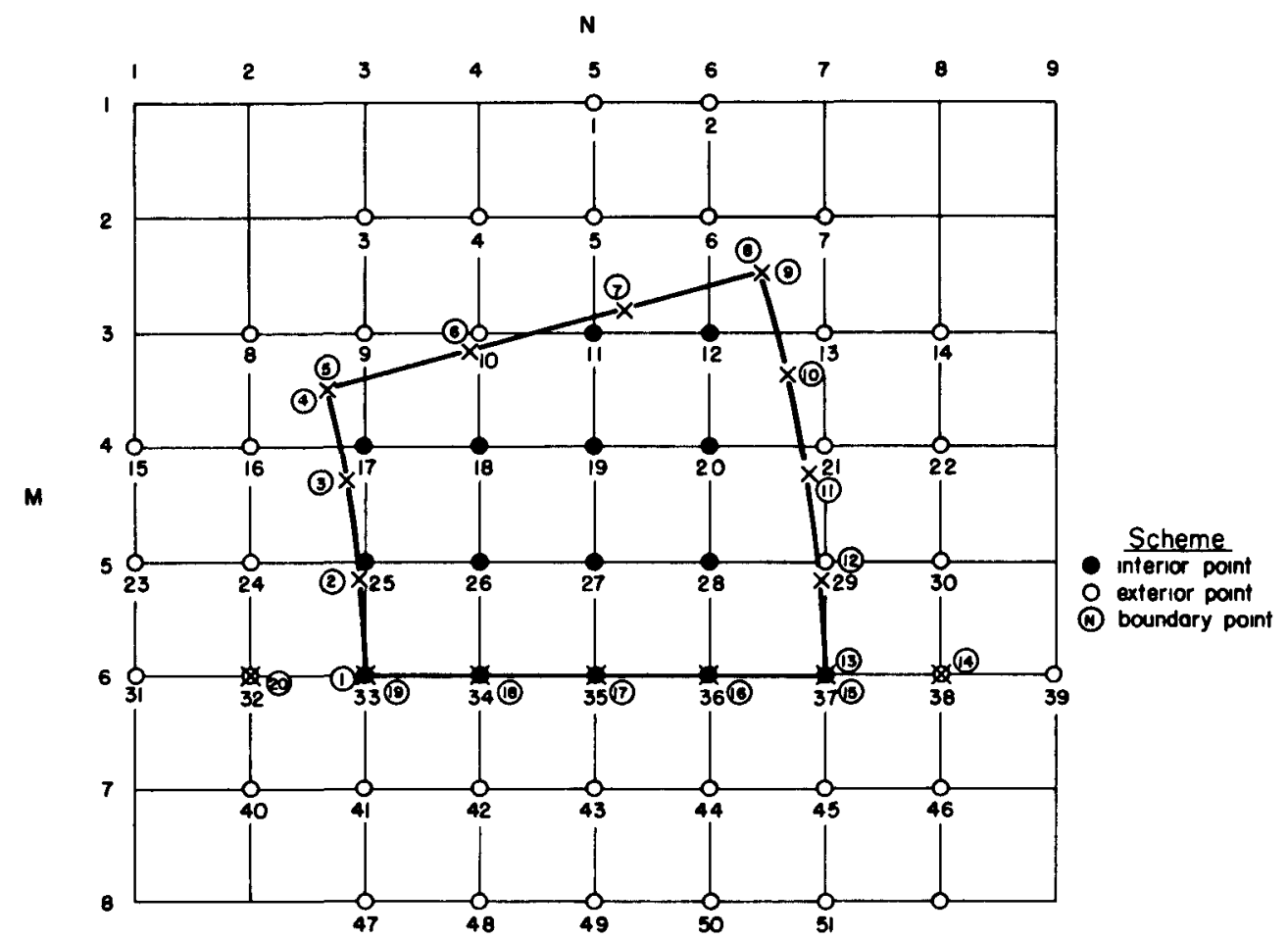

FIGURE 1. MESH WORK FOR SAMPLE PROBLEM (15-DEG SLICE OF ANNULUS)

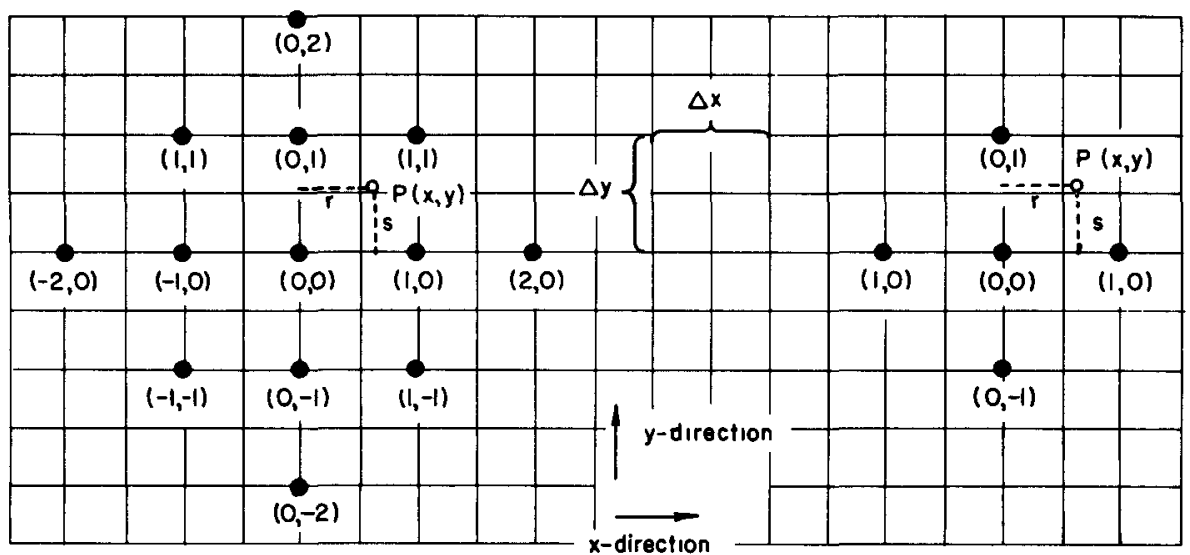

a Biharmonic Arroy

b. Laplacian Arroy

If $(0,0)$ Point is Located of $\left(x_{0}, y_{0}\right)$ Then for $P(x, y)\left\{\begin{array}{l}r=\frac{x-x_{0}}{\Delta x} \\ s=\frac{y-y_{0}}{\Delta y}\end{array}\right.$

A-33156 
For the stress problem the 13-point biharmonic array is used which is associated with the following interpolation formula

$$
\begin{aligned}
& \phi(r, s)= \phi(0,0)+\frac{r}{2}\left[\phi(1,0)-\phi(-1,0)+\frac{s}{2}[\phi(0,1)-\phi(0,-1)]\right. \\
&+\frac{r^{2}}{2}[\phi(1,0)+\phi(-1,0)-2 \phi(0,0)] \\
&+\frac{s^{2}}{2}[\phi(0,1)+\phi(0,-1)-2 \phi(0,0)] \\
&+\frac{r s}{4}[\phi(1,1)+\phi(-1,-1)-\phi(-1,1)-\phi(1,-1)] \\
&+\frac{r\left(r^{2}-1\right)}{12}[\phi(2,0)-2 \phi(1,0)-\phi(-2,0)+2 \phi(-1,0)] \\
&+\frac{\left.s^{2}-1\right)}{12}[\phi(0,2)-2 \phi(0,1)-\phi(0,-2)+2 \phi(0,-1)] \\
&+\frac{r s^{2}}{4}[\phi(1,1)+\phi(1,-1)-2 \phi(1,0)-\phi(-1,-1)-\phi(-1,1)+2 \phi(-1,0)] \\
&+\frac{r^{2} s}{4}[\phi(1,1)+\phi(-1,1)-2 \phi(0,1)-\phi(1,-1)-\phi(-1,-1)+2 \phi(0,-1)] \\
&+\frac{r^{2}\left(r^{2}-1\right)}{24}[6 \phi(0,0)-4 \phi(1,0)-4 \phi(-1,0)+\phi(2,0)+\phi(-2,0)] \\
&+\frac{s^{2}\left(s^{2}-1\right)}{24}[6 \phi(0,0)-4 \phi(0,1)-4 \phi(0,-1)+\phi(0,2)+\phi(0,-2)] \\
&+\frac{r^{2} s^{2}}{4}[\phi(1,1)+\phi(-1,1)+\phi(1,-1)+\phi(-1,-1) \\
&-2 \phi(0,1)-2 \phi(1,0)-2 \phi(-1,0) \\
&-2 \phi(0,-1)+4 \phi(0,0)]-
\end{aligned}
$$

It will be noted that both interpolation formulas give the correct value for the function when $(r, s)$ corresponds to one of the mesh points of the appropriate array. This property of the interpolation formulas demonstrates one of several superior features of interpolation formulas as contrasted to the frequently used two-dimensional Taylor series expansions.

Equations (20) and (21) are the fundamental formulas used throughout the rest of this report to obtain equations involving the point values of the functions at the mesh points. Since the mesh length is defined to be unity in these formulas, the unit of length $\mathrm{c}$ in the dimensionless equations is chosen to be the physical length of the mesh. This choice simplifies the numerical equations which follow but does not give a convenient dimensionless form to the final results. However, the final results can be put into a more convenient dimensionless form by multiplying the dimensionless temperature function, say, by $\left(\frac{c}{a}\right)^{2}$ where a is perhaps the radius of the hole. In this way the dimensionless forms are made independent of the particular mesh spacing used. 
Numerical Equations

The numerical equations to be solved are obtained by using the interpolation formulas in the differential and integral equations which define the problem. This is done in such a way that the number of independent linear algebraic equations involving the point values of the functions equals the number of point values of the function which are determined. For example, in Figure 1 there are 51 point values to be determined and, hence, 51 independent equations must be obtained. These equations will be linear algebraic equations because the interpolation formulas, differential equations, and integration schemes used are linear. It should be noted, however, that there is considerable arbitrariness in selecting the equations, and a certain amount of caution is necessary to avoid the use of dependent equations. The best guide in this regard is probably a combination of physical intuition and mathematical insight.

Probably the best procedure to be followed in writing the appropriate number of equations is to associate every mesh point with a specific equation. The equation selected should involve the point value of the function as strongly as possible. This last requirement while not imperative will facilitate the numerical solution and will help in avoiding dependent equations.

The interior points present no special problems, for the natural choice is to associate the equation $\nabla^{4} \phi=1$ or $\nabla^{2} \mathrm{~T}=-1$ with each interior point. For example, referring to Figure 1, the eleventh equation for the stress problem would read

$$
\begin{aligned}
\left(\nabla^{4} \phi\right)_{11}= & 20 \phi(11)-8[\phi(12)+\phi(5)+\phi(10)+\phi(19)] \\
& +2[\phi(6)+\phi(4)+\phi(18)+\phi(20)] \\
& +[\phi(13)+\phi(1)+\phi(9)+\phi(27)]=1 .
\end{aligned}
$$

The eleventh equation for the temperature problem would read

$$
\left(\nabla^{2} T\right)_{11}=-4 T(11)+T(12)+T(5)+T(10)+T(19)=-1 \text {. }
$$

Note that these equations were obtained by applying the differential operators at Point 11 to the interpolation formulas.

The exterior points, however, do present special problems. The general procedure is to associate an exterior point with a nearby boundary point (not necessarily a mesh point) and a boundary equation appropriate to that boundary. The boundary equation is obtained by using an interpolation formula centered at an interior point near the boundary point and performing the required operations on it. For example, referring to Figure 1, Mesh Point 6 is associated with Boundary Point 8 at the corner. Boundary Point 8 in turn is associated with interior Mesh Point 12 where an interpolation formula can be written. Thus, for the temperature problem the boundary equation $\frac{\partial \mathrm{T}}{\partial \mathrm{n}}=0$ is written at Boundary Point 8 using an interpolation formula centered at Mesh Point 12. Or, in detail, the equation for Mesh Point 6 for the temperature problem is obtained as follows: 


$$
\begin{aligned}
\left(\frac{\partial T}{\partial n}\right)_{r, s}= & n_{x} \frac{\partial T}{\partial r}+n_{y} \frac{\partial T}{\partial s}=0 \\
= & -\sin 15^{\circ}\left\{\frac{1}{2}[T(13)-T(11)]+r[R(13)+T(11)-2 T(6)]\right\} \\
& +\cos 15^{\circ}\left\{\frac{1}{2}[T(6)-T(20)]+s[T(6)+T(20)-2 T(6)]\right\}=0,
\end{aligned}
$$

where $(r, s)$ are the coordinates of Boundary Point 8 referred to interior Mesh Point 12 as origin. On the other hand, since Mesh Point 2 is not involved in the Laplacian array about any interior mesh point, this mesh point is eliminated by simply writing the equation: $\mathrm{T}(2)=0$.

For the stress problem Mesh Point 2 is associated with the equation $\frac{\partial \nabla^{2} \phi}{\partial n}=0$, written at Boundary Point 8, while Mesh Point 6 is associated with the equation $\frac{\partial \phi}{\partial n}=0$, written also at Boundary Point 8. Thus, two independent boundary conditions are applied to the same boundary point and referred to the same interior mesh point as an interpolation center.

In this fashion the required number of independent equations are obtained which can then be used to determine the point values of the temperature and the stress function at the mesh points. The stress-function solution in turn can be used with the interpolation formulas to determine the stress values at any desired point.

A detailed specification of the example of Figure 1 can be found in Appendix A. However, several important features might be mentioned here. A study of the problem reveals that the stress function is arbitrary only to a constant. Accordingly, the equation for Mesh Point 39 is arbitrarily taken to be $\phi(39)=0$. At the inside radius an integral equation [Equation (18)] must be written; this equation is arbitrarily associated with Point 32. Also, it may be noted that particular care must be exercised at the corners to avoid dependent equations. For example at Boundary Point 8 the equation $\frac{\partial \phi}{\partial n}=0$ is written. At Boundary Point 9 (the same point but with a different normal vector) the equations $\frac{\partial \phi}{\partial x}=0$ and $\frac{\partial \phi}{\partial y}=0$ could be written and would satisfy the boundary conditions on this boundary. However, $\frac{\partial \phi}{\partial n}, \frac{\partial \phi}{\partial x}$, and $\frac{\partial \phi}{\partial y}$ are not independent at the same point and, hence, such a procedure will lead to a dependent equation which cannot be used.

The line integrals encountered are reduced to linear algebraic equations by applying a simple trapezoidal rule to integrand values obtained at equally spaced boundary points. The integrands are evaluated by means of the usual interpolation formulas. The average temperature over the cross section of the body which is required in Equation (8) is obtained by a crude arithmetic average of the temperature values at the interior mesh points. 
Hence, as a result of the procedures described above the problem of Figure 1 has been reduced to solving 51 linea $r$ algebraic equations for first the temperature and then the stress functions. Although this size problem can be solved directly by a matrix inversion this approach becomes impossible as the number of equations becomes large. Hence, iterative types of solution are of interest for their application to the stress problem. The following section of the report describes an iterative procedure studied and used in this connection.

Before taking up the iterative procedure it should be mentioned that the preparation of the coefficient matrix for a large-scale problem can be a formidable task in itself. Consequently, all the numerical schemes for generating the coefficient matrix elements and processing the solutions have been coded for machine computation. In this way a minimum amount of hand-prepared input data is used. This not only simplifies the setup of the problem but reduces the possibility for errors. The hand-prepared input required for a problem setup is described in Appendix A.

\section{ITERATIVE METHOD OF SOLUTION}

As already mentioned the numerical method of solution reduces to the solution of a number of simultaneous linear algebraic equations. Consider, therefore, such a system of equations given by

$$
\mathrm{A} \phi=\mathrm{a}
$$

where

$$
\phi=\left(\begin{array}{c}
\phi_{1} \\
\phi_{2} \\
\phi_{3} \\
\cdot \\
\cdot \\
\phi_{\mathrm{R}}
\end{array}\right), \quad \mathrm{a}=\left(\begin{array}{c}
\mathrm{a}_{1} \\
\mathrm{a}_{2} \\
\mathrm{a}_{3} \\
\cdot \\
\cdot \\
\mathrm{a}_{\mathrm{R}}
\end{array}\right)
$$

and $A$ is a nonsingular RxR matrix. The $\phi_{1}, \phi_{2}, \ldots$ etc. are the unknowns to be determined.

An iteration scheme for solving Equation (22) can be employed. Of the many iteration schemes, the extrapolated Liebmann process has a number of advantages for machine computation. (4) This method will be described and related to a minimization process for iterating on Equation (22); this connection easily establishes the convergence of the extrapolated Liebmann process applied to an equation related to Equation (22): 


$$
A^{T} A=A^{T},
$$

or

$$
\mathrm{B} \phi=\mathrm{b},
$$

where

$$
\begin{aligned}
& \mathrm{B}=\mathrm{A}^{\mathrm{T}} \mathrm{A} \\
& \mathrm{b}=\mathrm{A}^{\mathrm{T}} \mathrm{a} .
\end{aligned}
$$

That is Equation (23) is simply obtained by multiplying Equation (22) by the transpose of matrix $A, A^{T}$. The matrix $B$ defined in this way is a positive definite matrix.

\section{Minimization Process for Iteration}

The minimization scheme described here is closely related to several other iteration schemes but apparently it has not appeared in the literature.

The residual vector $r$ is defined by

$$
\mathrm{r}=\mathrm{A} \phi-\mathrm{a},
$$

and the length squared of $r$ is given by

$$
|r|^{2}=r \cdot r=|A \phi-a|^{2} .
$$

The iteration scheme assumes that there is an estimate for the vector $\phi$ :

$$
\phi^{\mathrm{n}}(\mathrm{s})=\left(\begin{array}{c}
\phi_{1}^{\mathrm{n}+1} \\
\phi_{2}^{\mathrm{n}+1} \\
\cdot \\
\cdot \\
\phi_{\mathrm{s}-1}^{\mathrm{n}+1} \\
\phi_{\mathrm{s}}^{\mathrm{n}} \\
\cdot \\
\cdot \\
\phi_{\mathrm{R}}^{\mathrm{n}}
\end{array}\right)
$$


That is, at this stage of the $\mathrm{n}^{\text {th }}$ iteration the elements $\phi_{1}, \phi_{2}, \ldots, \phi_{\mathrm{S}-1}$ have been iterated, but the elements $\phi_{\mathrm{s}}, \phi_{\mathrm{s}+1}, \ldots, \phi_{\mathrm{R}}$ have not been iterated as yet. Then it is desired to determine $\phi_{S}^{\mathrm{n}+1}$ in such a way that $|r|^{2}$ is minimized. To determine the way this choice must be made $\phi^{n}(s+1)$ is put into Equation (25) for $\phi$ and it is required that

$$
\frac{\partial|r|^{2}}{\partial \phi_{s}^{n+1}}=0
$$

which gives

$$
\frac{\partial|r|^{2}}{\partial \phi_{\mathbf{s}}^{n+1}}=2 r \cdot \frac{\partial r}{\partial \phi_{s}^{n+1}}=2(A \phi-a) \cdot A \frac{\partial \phi}{\partial \phi_{s}^{n+1}}=0 .
$$

However,

$$
\begin{aligned}
\frac{\partial \phi_{r}^{\mathrm{n}}(\mathrm{s}+1)}{\partial \phi_{\mathrm{s}}^{\mathrm{n}+1}} & =0, \quad r \neq s, \\
& =1, \quad r=s,
\end{aligned}
$$

and, therefore,

$$
\sum_{k}\left[A \phi^{n}(s+1)-a\right]_{k} \cdot A_{k s}=0
$$

or in detail,

$$
\sum_{k}\left\{\sum_{l} A_{k l} \phi_{l}^{n}(s+1)-a_{k}\right\} A_{k s}=0,
$$

or using the notation for the residual vector corresponding to $\phi_{l}^{\mathrm{n}}(s+1)$,

$$
\sum_{\mathrm{k}} \mathrm{r}_{\mathrm{k}}^{\mathrm{n}}(\mathrm{s}+1) \mathrm{A}_{\mathrm{ks}}=0
$$

where

$$
r_{k}^{n}(s+1)=\sum_{\ell} A_{k \ell} \phi_{\ell}^{n}(s+1)-a_{k} .
$$

An expression for $\phi_{s}^{\mathrm{n}+1}$ can be obtained by noting that

$$
r_{k}^{n}(s+1)=r_{k}^{n}(s)+A_{k s}\left[\phi_{s}^{n+1}-\phi_{s}^{n}\right]
$$

Putting the last expression into Equation (25) gives finally

$$
\phi_{s}^{n+1}=\phi_{s}^{n}-\frac{1}{\sum_{k} A_{k s}^{2}} \sum_{k} A_{k s} r_{k}^{n}(s) \text {. }
$$


This, then, is the new value of $\phi_{\mathbf{S}}$ which minimizes the square of the residual vector. Further, since the vectors

$$
\left(\begin{array}{l}
A_{1 s} \\
A_{2 s} \\
\cdot
\end{array}\right)
$$

are linearly independent and the minimization process will be complete only if Equation (26) is satisfied for all values of $\mathrm{s}$, it follows that there exists only the minimum given by the solution $A \phi=c$. Hence, the iteration scheme will always converge to the true solution.

\section{The Extrapolated Liebmann Process}

There exists an interesting relationship between the minimization process just described and the extrapolated Liebmann process applied to the transposed system $\mathrm{A}^{\mathrm{T}} \mathrm{A} \phi=\mathrm{A}^{\mathrm{T}} \mathrm{a}$.

Thus, consider first the system

$$
\mathrm{A} \phi=\mathrm{a}
$$

The extrapolated Liebmann iteration scheme for the transposed system $\left(\mathrm{B}_{\mathrm{SS}} \neq 0\right)$ is

$$
\phi_{s}^{n+1}=\phi_{s}^{n}-\frac{\alpha}{B_{s s}}\left\{\sum_{l} B_{s l} \phi_{l}^{n}(s)-b_{s}\right\}
$$

or

$$
\phi_{s}^{n+1}=\phi_{s}^{n}-\frac{\alpha}{\sum_{k} A_{k s}^{2}}\left\{\sum_{k} A_{k s} r_{k}^{n}(s)\right\} .
$$

Thus, Equation (29) differs only from Equation (28) in that $\alpha$ appears in Equation (29). This significant result apparently has not appeared in the Iiterature. The question naturally arises as to what choices of $\alpha$ in Equation (29) will assure convergence. This question is easily answered by considering the residual vector change during the iteration. It is easily demonstrated that the square of the residual vector will never increase if $0<\alpha<2$. It suffices to observe that 


$$
\begin{aligned}
\delta & =\left|r^{n}(s+1)\right|^{2}-\left|r^{n}(s)\right|^{2} \\
& =\left|r^{n}(s)+A\left\{\phi^{n}(s+1)-\phi^{n}(s)\right\}\right| 2-\left|r^{n}(s)\right|^{2} \\
& =2 r^{n}(s) \cdot A\left\{\phi^{n}(s+1)-\phi^{n}(s)\right\}+\left[A\left\{\phi^{n}(s+1)-\phi^{n}(s)\right\}\right]^{2} \\
& =2 \sum_{k} r_{k}^{n}(s) A_{k s}\left\{\phi_{s}^{n+1}-\phi_{s}^{n}\right\}+\sum_{k s}\left\{\phi_{s}^{n+1}-\phi_{s}^{n}\right\}^{2} \\
& \left.=-2 \alpha \frac{A_{k s}^{2}}{k} \sum_{k s} r_{k}^{n}(s)\right\}^{2} \\
& \left.\left\{\sum_{k} A_{k s} r_{k}^{n}(s)\right\}_{k s} r_{k}^{n}(s)\right\}^{2} \\
& =\left(-2 \alpha+\alpha^{2}\right)
\end{aligned}
$$

Clearly for $0<\alpha<2$ the square of the residual vector will never increase at any step of the iteration scheme.

Another question of importance is the best choice of $\alpha$. At each step $\alpha=1$ gives the greatest decrease in the square of the residual vector; however, some other choice of $\alpha$ may well give a larger decrease after a number of iterations than the choice $\alpha=1$. This question of the optimum choice of $\alpha$ is a complex one. In general, there is little to be done other than experiment with different $\alpha$ choices to determine the best $\alpha$ choice for a given problem. To be sure for special matrices something more can be said about the optimum choice of $\alpha$ but these are special cases. (5)

The iteration scheme given by Equation (29) can be performed rather efficiently by a high-speed computer. Thus, define

$$
\begin{gathered}
\mu_{\mathrm{s}}=\frac{1}{\mathrm{~B}_{\mathrm{ss}}}, \\
\mathrm{r}_{\mathrm{k}}^{\mathrm{n}}(\mathrm{s})=\sum_{\ell} \mathrm{A}_{\mathrm{k} \ell} \phi_{\ell}^{\mathrm{n}}(\mathrm{s})-\mathrm{a}_{\mathrm{k}}, \\
\delta \phi_{\mathrm{s}}^{\mathrm{n}}=\phi_{\mathrm{s}}^{\mathrm{n}+1}-\phi_{\mathrm{s}}^{\mathrm{n}} .
\end{gathered}
$$


Then

$$
\begin{gathered}
\delta \phi_{s}^{n}=-\alpha \mu_{s} \sum_{k} A_{k s} r_{k}^{n}(s), \quad 1 \leq s \leq R, \\
r_{k}^{n}(s)=r_{k}^{n}(s-1)+A_{k, s-1} \delta \phi_{s-1}^{n}, \quad 2 \leq s \leq R+1, \\
r_{k}^{n}(1)=r_{k}^{n-1}(R+1) .
\end{gathered}
$$

The iteration starts with an estimate for $\phi_{s}^{1}, s=1, \ldots, R$. From this $r_{k}^{1}(1)$ is calculated by Equation (31). Then $\delta \phi_{1}^{l}$ is calculated from Equation (33). Then $\mathrm{r}_{\mathrm{k}}(2)$ is calculated from Equation (34). Then $\delta \phi_{2}^{1}$ is calculated from Equation (33). This continues until $r_{k}^{1}(R+1)$ is calculated by Equation (34). Then by Equation (35) the second iteration cycle is started and the process is repeated. The iteration is stopped when the residual vector has become small enough. This procedure reduces the computation considerably over say computing each $r_{k}^{n}(s)$ by Equation (31) directly each time.

The iteration routine described by Equations (30) through (35) has been coded for computation on the IBM-704. The details of the code will be found in Appendix A. Some of the results of the iteration scheme will be described in a later section of the report.

\section{Terminal Block Extrapolation}

The iteration routine described by Equations (30) through (35) may be improved by a process which might be called "terminal block extrapolation". This process is carried out by adding to the most recently computed vector $\phi$ ntl lobtained at the end of the $\mathrm{n}^{\text {th }}$ iteration cycle) a correction proportional to the $\delta \phi^{\mathrm{n}}$ vector. Thus,

$$
\phi(K)=\phi^{\mathrm{n}+1}+K \delta \phi^{\mathrm{n}}
$$

The factor $\mathrm{K}$ can now be determined such that $\phi(\mathrm{K})$ reduces the residual as much as possible. Thus, if

$$
\begin{gathered}
r(K)=A \phi(K)-a \\
r=A \phi^{n+1}-a \\
\delta r=A \delta \phi^{n}
\end{gathered}
$$

then

$$
r^{2}(K)=r^{2}+2 K r \cdot \delta r+K^{2}(\delta r)^{2}
$$


Clearly $r^{2}(K)$ is minimized if

$$
K=-\frac{r \cdot \delta r}{(\delta r)^{2}}
$$

and, since $r \cdot \delta x<0, K>0$. Using this optimum choice in the expression for $\mathfrak{r}^{2}(K)$ gives

$$
\begin{aligned}
r^{2}(K) & =r^{2}-\frac{(r \cdot \delta r)^{2}}{(\delta r)^{2}}, \\
& =r^{2}\left(1-\cos ^{2} \theta\right), \\
r^{2}(K) & =r^{2} \sin ^{2} \theta,
\end{aligned}
$$

where

$$
\cos \theta=\frac{r \cdot \delta r}{\sqrt{\left(r^{2}\right)(\delta r)^{2}}}=\frac{r \cdot \delta r}{|r||\delta r|} .
$$

Thus, this important result indicates that the terminal block extrapolation with the optimum value for $\mathrm{K}$ will reduce the residual vector significantly if the vectors $r$ and $\delta \mathrm{r}$ are nearly parallel. By virtue of the fact that $\delta r$ is obtained in such a way that $r$ is shortened $\delta r$ cannot be perpendicular to $r$ but rather the minimization process tends to make $\delta r$ and $r$ parallel. Hence, a combination of the minimization iteration process and the terminal block extrapolation process appears to be a very promising method for solving linear algebraic systems of equations.

As an indication of the important gains in convergence which can be obtained a simple problem was solved by both the iteration scheme of Equation (29) and the iteration scheme augmented by terminal block extrapolation. The equation to be solved was:

$$
\left(\begin{array}{rrr}
-2 & 1 & 0 \\
1 & -2 & 1 \\
0 & 1 & -2
\end{array}\right)\left(\begin{array}{l}
\phi_{1} \\
\phi_{2} \\
\phi_{3}
\end{array}\right)=\left(\begin{array}{l}
-1 \\
-1 \\
-1
\end{array}\right)
$$

with the solution $\phi_{1}=\phi_{3}=1.5$ and $\phi_{2}=2.0$. Table 1 shows the residual vector squared for every other iteration and the $\mathrm{K}$-value for the extrapolation for every iteration. The solution vectors after 24 iterations in the two cases were:

(1) No extrapolation

$$
\begin{aligned}
& \phi_{1}=1.5020347 \\
& \phi_{2}=2.0026662 \\
& \phi_{3}=1.5017260
\end{aligned}
$$


TABLE 1. COMPARISON OF RESIDUALS WITH AND WITHOUT EXTRAPOLATION

\begin{tabular}{|c|c|c|c|}
\hline & $\begin{array}{c}\text { No Extrapolation } \\
\text { Square of } \\
\text { Residual Vector }\end{array}$ & $\begin{array}{l}\text { Extrapolation } \\
\text { Square of } \\
\text { Residual Vector }\end{array}$ & $\begin{array}{c}\text { Extrapolation } \\
\text { Factor } \\
\text { K }\end{array}$ \\
\hline 0 & $8.24 \times 10^{-2}$ & $8.24 \times 10^{-2}$ & \\
\hline 2 & $1.41 \times 10^{-3}$ & $1.36 \times 10^{-3}$ & $\begin{array}{c}0.10 \\
-0.025\end{array}$ \\
\hline 4 & $8.91 \times 10^{-4}$ & $3.93 \times 10^{-4}$ & $\begin{array}{l}1.33 \\
1.35\end{array}$ \\
\hline 6 & $5.34 \times 10^{-4}$ & $4.10 \times 10^{-5}$ & $\begin{array}{l}0.019 \\
6.78\end{array}$ \\
\hline 8 & $3.18 \times 10^{-4}$ & $4.92 \times 10^{-7}$ & $\begin{array}{l}0.17 \\
0.51\end{array}$ \\
\hline 10 & $1.89 \times 10^{-4}$ & $8.18 \times 10^{-8}$ & $\begin{array}{c}3.14 \\
-0.081\end{array}$ \\
\hline 12 & $1.12 \times 10^{-4}$ & $1.07 \times 10^{-8}$ & $\begin{array}{l}3.63 \\
0.44\end{array}$ \\
\hline 14 & $6.73 \times 10^{-5}$ & $8.62 \times 10^{-10}$ & $\begin{array}{l}0.19 \\
5.60\end{array}$ \\
\hline 16 & $4.01 \times 10^{-5}$ & $1.62 \times 10^{-10}$ & $\begin{array}{c}-0.083 \\
2.02\end{array}$ \\
\hline 18 & $2.39 \times 10^{-5}$ & $4.89 \times 10^{-11}$ & $\begin{array}{l}0.74 \\
0.048\end{array}$ \\
\hline 20 & $1.42 \times 10^{-5}$ & $2.53 \times 10^{-14}$ & $\begin{array}{r}7.02 \\
-0.12\end{array}$ \\
\hline 22 & $8.48 \times 10^{-6}$ & $9.29 \times 10^{-15}$ & $\begin{array}{l}0.96 \\
0.60\end{array}$ \\
\hline 24 & $5.06 \times 10^{-6}$ & $8.15 \times 10^{-16}$ & $\begin{array}{l}0.05 \\
5.43\end{array}$ \\
\hline
\end{tabular}


(2) Extrapolation every iteration

$$
\begin{aligned}
& \phi_{1}=1.5000001 \\
& \phi_{2}=2.0000005 \\
& \phi_{3}=1.5000003 .
\end{aligned}
$$

In each case the initial guess was

$$
\begin{aligned}
& \phi_{1}=1.4000000 \\
& \phi_{2}=1.9800000 \\
& \phi_{3}=1.6000000 .
\end{aligned}
$$

It will be noted from Table 1 that the extrapolation process has accelerated the convergence immensely. It will also be noted from the solution vectors after 24 iterations that the accuracy is much better in the case of the extrapolation case. In fact the small errors in the last digit are apparently round-off error accumulation.

It is also of interest to compare the terminal extrapolation procedure with the iteration procedure using an optimum value for $\alpha$ in Equation (29). By experiment an optimum value of $\alpha=1.49$ was found. After 25 iterations the residual vector squared was $2.89 \times 10^{-15}$ compared to a value of $2.48 \times 10^{-16}$ for the terminal block extrapolation case. Hence, the terminal block extrapolation was superior in this case to using an optimum value for $\alpha$. Of course, in most cases the optimum value for $\alpha$ will not be known, and this points out the superiority of the terminal block extrapolation since no additional information about the system is required. To be sure some additional computation is required to carry out the terminal block extrapolation, but this is a small amount compared to the computations required for one iteration cycle in a large problem.

Studies of a similar nature must be carried out on larger systems to determine the usefulness of the terminal block extrapolation process on large-scale systems. The effect of extrapolating every $n^{\text {th }}$ iteration must also be studied.

\section{NUMERICAL RESULTS}

\section{Preliminary Studies}

In this part of the report some results are described which were obtained by applying the methods presented above. First, the sample problem of Figure 1 will be considered, and then some results for other geometries will be reviewed.

The simple annulus problem of Figure 1 can be solved analytically. In addition, the 51 equations which result from the numerical formulation can be solved directly. 
Thus, the validity of the numerical approximations can be determined by comparing the results of these two methods of solution. The results from the iterative method of solution can then be compared with the results of the other solutions. In this way the effect of a smaller mesh spacing can also be studied.

The analytical solutions for the dimensionless temperature function and stress function(l) for the annulus of Figure 1 are as follows:

$$
T(r)=\frac{b^{2}-a^{2}}{2 a \beta}+\frac{b^{2}}{4}\left\{\ln \frac{r^{2}}{a^{2}}-\frac{r^{2}-a^{2}}{b^{2}}\right\},
$$

where

$$
\begin{aligned}
& a=10, \text { inside radius } \\
& b=14, \text { outside radius } \\
& r=\text { dimensionles s radius } \\
& \beta=0.024,
\end{aligned}
$$

and

$$
\phi(r)=A \log r+B r^{2} \log r+C r^{2}+D+\frac{r^{2}}{64}
$$

where

$$
\begin{aligned}
& A=-2141.1244 \\
& B=-24.500000 \\
& C=76.243957 \\
& D=2779.2375 .
\end{aligned}
$$

The stress function given by Equation (37) is based on the condition of $\phi=0$, at $r=b$.

The same problem was then solved using the numerical procedures described in this report, first, by a direct matrix inversion and then by the iteration method. A comparison of the results from the various procedures is given in Tables 2 through 6; however, these results need some further explanation. The iteration solution was started with an initial guess rather close to the correct answer. The initial guesses used are shown in parentheses next to the iteration values. Then an iteration solution was performed with the mesh spacing halved. The results of this solution at the mesh points of the coarser mesh are also shown in the tables.

First, it may be noted from the matrix-inversion results given in the tables that even for a coarse mesh the numerical schemes give rather good results for the solution of the problem. In the case of the temperature function there is about a 0.02 difference between the analytical and the matrix-inversion solution. This is a rather uniform difference and it is not surprising since the temperature level enters the problem in a rather weak connection. The iteration results are not too meaningful if only the 
TABLE 2. TEMPERATURE FUNCTION

$$
\frac{T(x, y)}{\frac{Q a^{2}}{k}}
$$

\begin{tabular}{|c|c|c|c|c|}
\hline \multirow{3}{*}{$\begin{array}{c}\text { Interior } \\
\text { Point }\end{array}$} & \multirow[b]{3}{*}{ Analytical } & \multicolumn{2}{|c|}{ Full Mesh } & \multirow{3}{*}{$\begin{array}{c}\text { Half Mesh, } \\
300 \\
\text { Iterations }\end{array}$} \\
\hline & & Matrix & 300 & \\
\hline & & Inversion & Iterations & \\
\hline 11 & 2.076 & 2.095 & $2.095(2.095)$ & $2.076(2.075)$ \\
\hline 12 & 2.087 & 2.107 & $2.106(2.110)$ & $2.088(2.085)$ \\
\hline 17 & 2.009 & 2.028 & $2.029(2.030)$ & $2.008(2.010)$ \\
\hline 18 & 2.047 & 2.066 & $2.066(2.065)$ & $2.046(2.045)$ \\
\hline 19 & 2.072 & 2.091 & $2.091(2.090)$ & $2.072(2.070)$ \\
\hline 20 & 2.086 & 2.105 & $2.105(2.105)$ & $2.086(2.085)$ \\
\hline 25 & 2.002 & 2.021 & $2.021(2.020)$ & $2.001(2.000)$ \\
\hline 26 & 2.042 & 2.061 & $2.062(2.060)$ & $2.042(2.040)$ \\
\hline 27 & 2.070 & 2.089 & $2.089(2.089)$ & $2.069(2.070)$ \\
\hline 28 & 2.085 & 2. 104 & $2.104(2.105)$ & $2.085(2.085)$ \\
\hline 33 & 2.000 & 2.019 & $2.019(2.020)$ & $2.000(2.000)$ \\
\hline 34 & 2.041 & 2.060 & $2.060(2.060)$ & $2.040(2.040)$ \\
\hline 35 & 2.070 & 2.088 & $2.088(2.090)$ & $2.068(2.070)$ \\
\hline 36 & 2.085 & 2. 104 & $2.104(2.105)$ & $2.085(2.085)$ \\
\hline 37 & 2.090 & 2. 109 & $2.109(2.110)$ & $2.090(2.090)$ \\
\hline
\end{tabular}


TABLE 3. STRESS FUNC TION

$$
\frac{\phi \times 10^{4}}{\frac{\alpha E}{1-\nu} \times \frac{Q a^{4}}{k}}
$$

\begin{tabular}{|c|c|c|c|c|}
\hline \multirow{3}{*}{$\begin{array}{l}\text { Interior } \\
\text { Point }\end{array}$} & \multirow[b]{3}{*}{ Analytical } & \multicolumn{2}{|c|}{ Full Mesh } & \multirow{3}{*}{$\begin{array}{l}\text { Half Mesh, } \\
\quad 300 \\
\text { Iterations }\end{array}$} \\
\hline & & Matrix & 300 & \\
\hline & & Inversion & Iterations & \\
\hline 11 & -3.371 & -3.531 & $-3.598(-3.5)$ & $-3.060(-3.0)$ \\
\hline 12 & -0.577 & -0.606 & $-0.631(-0.5)$ & $-0.491(-0.5)$ \\
\hline 17 & -11.458 & -11.951 & $-11.937(-12.0)$ & $-11.316(-11.0)$ \\
\hline 18 & -8.493 & -8.881 & $-8.931(-9.0)$ & $-8.303(-8.5)$ \\
\hline 19 & -4.181 & -4.389 & $-4.455(-4.5)$ & $-4.387(-4.5)$ \\
\hline 20 & -0.949 & -1.003 & $-1.027(-1.0)$ & $-9.971(-1.0)$ \\
\hline 25 & -11.566 & -12.066 & $-12.035(-12.0)$ & $-11.427(-11.5)$ \\
\hline 26 & -9.051 & -9.468 & $-9.502(-9.5)$ & $-8.965(-9.0)$ \\
\hline 27 & -4.699 & -4.934 & $-4.998(-5.0)$ & $-4.660(-4.5)$ \\
\hline 28 & -1.227 & -1.284 & $-1.308(-1.5)$ & $-1.208(-1.0)$ \\
\hline 33 & -11.572 & -12.074 & $-12.045(-12.0)$ & $-11.434(-11.5)$ \\
\hline 34 & -9.234 & -9.654 & $-9.680(-9.5)$ & $-9.149(-9.0)$ \\
\hline 35 & -4.874 & -5.114 & $-5.176(-5.0)$ & $-4.832(-5.0)$ \\
\hline 36 & -1.312 & -1.368 & $-1.399(-1.5)$ & $-1.293(-1.0)$ \\
\hline 37 & 0 & 0 & $(0)$ & $(0)$ \\
\hline
\end{tabular}


TABLE 4, STRESS COMPONENT

$$
\frac{\sigma_{\mathrm{x}} \times 10^{2}}{\frac{\alpha E}{1-\nu} \frac{\sigma^{2}}{k}}
$$

\begin{tabular}{|c|c|c|c|c|}
\hline \multirow{3}{*}{$\begin{array}{l}\text { Interior } \\
\text { Point }\end{array}$} & \multirow[b]{3}{*}{ Analytical } & \multicolumn{2}{|c|}{ Full Mesh } & \multirow{3}{*}{$\begin{array}{c}\text { Half Mesh, } \\
300 \\
\text { Iterations }\end{array}$} \\
\hline & & Matrix & 300 & \\
\hline & & Inversion & Iterations & \\
\hline 11 & 0.199 & 0.208 & 0.202 & -0.098 \\
\hline 12 & -0.006 & 0.010 & -0.014 & -0.122 \\
\hline 17 & 0.312 & 0.346 & 0.376 & 0.389 \\
\hline 18 & 0.406 & 0.439 & 0.441 & 0.494 \\
\hline 19 & 0.297 & 0.319 & 0.318 & 0.379 \\
\hline 20 & 0.108 & 0.122 & 0.118 & 0.135 \\
\hline 25 & 0.090 & 0.095 & 0.073 & 0.071 \\
\hline 26 & 0.375 & 0.399 & 0.393 & 0.485 \\
\hline 27 & 0.345 & 0.371 & 0.371 & 0.484 \\
\hline 28 & 0.176 & 0.204 & 0.198 & 0.231 \\
\hline 33 & 0 & 0.002 & 0.008 & -0.013 \\
\hline 34 & 0.358 & 0.369 & 0.347 & 0.351 \\
\hline 35 & 0.359 & 0.358 & 0.354 & 0.321 \\
\hline 36 & 0.199 & 0.165 & 0.181 & 0.164 \\
\hline 37 & 0 & 0 & 0.013 & 0.001 \\
\hline
\end{tabular}


TABLE 5. STRESS COMPONENT

$$
\frac{\sigma_{y} \times 10^{2}}{\frac{\alpha E}{1-\nu} \frac{Q^{2}}{k}}
$$

\begin{tabular}{lcccc}
\hline \multirow{2}{*}{$\begin{array}{c}\text { Interior } \\
\text { Point }\end{array}$} & Analytical & $\begin{array}{c}\text { Matrix } \\
\text { Inversion }\end{array}$ & $\begin{array}{c}300 \\
\text { Iterations }\end{array}$ & $\begin{array}{c}\text { Half Mesh, } \\
300 \\
\text { Iterations }\end{array}$ \\
\cline { 4 - 5 } 11 & -1.465 & -1.492 & -1.440 & -1.739 \\
12 & -2.426 & -2.546 & -2.566 & -2.999 \\
17 & 5.089 & 5.287 & 5.158 & -5.238 \\
18 & 1.233 & 1.298 & 1.360 & -1.101 \\
19 & -1.184 & -1.208 & -1.142 & -1.262 \\
20 & -2.393 & -2.498 & -2.519 & -2.313 \\
25 & 5.995 & 6.225 & 6.037 & 5.787 \\
26 & 1.704 & 1.798 & 1.850 & 1.727 \\
27 & -0.979 & -0.994 & -0.916 & -0.963 \\
28 & -2.354 & -2.469 & -2.478 & -2.336 \\
33 & 6.322 & 6.552 & 6.286 & 6.142 \\
34 & 1.874 & 1.980 & 2.022 & 1.912 \\
35 & -0.904 & -0.907 & -0.830 & -0.892 \\
36 & -2.338 & -2.453 & -2.455 & -2.347 \\
37 & -2.652 & -3.000 & -3.064 & -2.573 \\
\hline \hline
\end{tabular}


TABLE 6. STRESS COMPONENT

$$
\frac{\tau_{\mathrm{xy}} \times 10^{2}}{\frac{\alpha \mathrm{E}}{1-\nu} \frac{\mathrm{Qa}^{2}}{\mathrm{k}}}
$$

\begin{tabular}{|c|c|c|c|c|}
\hline \multirow{3}{*}{$\begin{array}{l}\text { Interior } \\
\text { Point }\end{array}$} & \multirow[b]{3}{*}{ Analytical } & \multicolumn{2}{|c|}{ Full Mesh } & \multirow{3}{*}{$\begin{array}{c}\text { Half Mesh, } \\
300 \\
\text { Iterations } \\
\end{array}$} \\
\hline & & Matrix & 300 & \\
\hline & & Inversion & Iterations & \\
\hline 11 & 0.529 & 0.414 & 0.412 & 0.406 \\
\hline 12 & 0.590 & 0.641 & 0.592 & 0.613 \\
\hline 17 & -0.995 & -1.094 & -1.052 & -1.108 \\
\hline 18 & -0.155 & -0.208 & -0.213 & -0.259 \\
\hline 19 & 0.254 & 0.232 & 0.225 & 0.311 \\
\hline 20 & 0.394 & 0.385 & 0.389 & 0.508 \\
\hline 25 & -0.596 & -0.648 & -0.595 & -0.554 \\
\hline 26 & -0.122 & -0.150 & -0.153 & -0.156 \\
\hline 27 & 0.111 & 0.102 & 0.094 & 0.136 \\
\hline 28 & 0.196 & 0.182 & 0.187 & 0.207 \\
\hline 33 & 0 & 0 & 0.000 & -0.001 \\
\hline 34 & 0 & 0 & 0.000 & 0.000 \\
\hline 35 & 0 & 0 & 0.000 & 0.000 \\
\hline 36 & 0 & 0 & 0.000 & 0.000 \\
\hline 37 & 0 & 0 & 0.000 & 0.000 \\
\hline
\end{tabular}


four-figure results displayed are considered. However, the iteration process has reduced the residuals considerably from the initial values given by the initial guess. Thus, a temperature function has been determined which is much smoother than the initially chosen function. The one-half-mesh results are also much closer to the analytical solution, but the initial values used are also much closer to the analytical solution.

In the case of the stress-function results shown in Table 3, the comparison between the analytical and matrix-inversion results is again good considering the coarse mesh employed. The iteration results are more meaningful here than in the temperature case. The comparison between the matrix-inversion and the 300-iteration results is rather good, and the results agree rather well with the analytical results. Again, a stress function has been generated by the iteration process which is much smoother than the initial guess. The same comments can be made about the stress components given in Tables 4 through 6, which were computed from the stress-function results. Comparing the half-mesh results with the full-mesh results, it is noted that the half-mesh results for the temperature and stress functions are nearer to the analytical solutions, in general. However, comparing the stress components it is noted that the full-mesh results are more accurate than the half-mesh results. The explanation of this is that the convergence of the half-mesh problem is slower than for the full-mesh problem, and, hence, after 300 iterations a "smoother" function is obtained in the coarse-mesh case. The stress components involve differential operations upon the stress function, and, hence, any lack of smoothness in the stress function will be evident in the stress components. This illustrates an important point: the best numerical procedure is a compromise between the requirement of a coarse mesh for better convergence and the requirement of a fine mesh for better accuracy in the interpolation formulas.

The iteration process used for this sample problem employed an acceleration factor of $\alpha=1$. Some studies were made with the sample problem which involved varying the value of $\alpha$. It was found that after several hundred iterations the choice $a=1$ reduces the residual about the same as or more than any other choice of $\alpha$. On the other hand if after several hundred iterations the $\alpha$-value is changed to a higher value, it is found that the residuals will decrease more rapidly than if the $\alpha=1$ value were maintained. Thus, it appears as if some procedure of changing the $\alpha$-value during the iteration might be superior to keeping the $\alpha$-value constant.

$\underline{\text { Fuel-Element Studies }}$

$\underline{\text { Single-Fole Hexagonal Element }}$

The fuel element shown in Figure 3 was also studied. In these cases analytical solutions are not available, and the large number of mesh points required make direct matrix-inversion methods undesirable if not impractical. The problem formulation is similar in every respect to the sample problem of the annulus. A symmetry section is isolated as shown in Figure 3 and the mesh work is constructed. The equations are formulated, the coefficient matrix is constructed, and the iterative process is applied to obtain an approximate solution.

Of particular significance is the maximum tensile stress. In the case of the annulus the maximum tensile stress occurs at the inside surface. In the case of the single-hole hexagonal elements the maximum tensile stress occurs at Point $\mathrm{A}$ in 
Figure 3. It is interesting to compare these maximum tensile stresses which occur in the hexagonal elements with the maximum tensile stresses which occur in "equivalent" annuli of the same inside radius and the same area as the single-hole hexagonal elements. Figure 4 shows a graph of the maximum tensile stress for the different types of elements for several values of the $\frac{t}{a}$ ratio, where $t$ is the length indicated in Figure 3 and $a$ is the hole radius. It will be noted that the maximum stresses are greater in the case of the hexagonal elements than the corresponding annular elements and that the deviation becomes more significant percentagewise as the $\frac{t}{a}$ value decreases. It should be remarked, however, that the single-hole hexagonal results shown in Figure 4 were obtained from the iteration-method results, and there may be some error incurred in using these approximate solutions.

Seven-Hole Hexagonal Element

The seven-hole hexagonal element shown in Figure 5 was also investigated. The symmetry section shown was subjected to the same type of analysis as described previously. The results for the maximum tensile stress are given in Figure 4. It will be noted that the results for the $\frac{t}{a}=0.5$ and $\frac{t}{a}=0.8$ cases lie rather close to the single-hole hexagonal results but that the $\frac{t}{a}=0.2$ is considerably different. While this difference may be real it should be mentioned that in the seven-hole $\frac{t}{a}=0.2$ case 339 mesh points were used which is larger than the other cases by more than 100 mesh points. As a result of this the convergence is poorer in this case than in the others. Further, the maximum tensile stress for the $\frac{t}{a}=0.5$ and $\frac{t}{a}=0.8$ seven-hole cases is in the $x-y$ plane and is found in the Region A noted in Figure 5. On the other hand, the maximum tensile stress for the $\frac{t}{a}=0.2$ seven-hole case is $\sigma_{z}$ and is located in the Region $B$ noted in Figure 5. An investigation of the $\frac{t}{a}=0.2$ case has shown that the temperatures determined are not reasonable and that probably the convergence is not satisfactory. The $\sigma_{z}$ depends upon the difference of the temperature at the point and the average temperature and, hence, $\sigma_{z}$ is quite sensitive to the temperature values. On the other hand, the other stresses in the problem are virtually independent of the temperature values. If the $\sigma_{z}$ stresses are disregarded in this case the maximum stress is then found to be in Region $A$ and to have a value much closer to the single-hole $\frac{t}{a}=0.2$ value.

Hence, it is concluded that the maximum tensile stress in the case of the sevenhole hexagonal element is essentially the same as that in the single-hole hexagonal element over the $\frac{t}{a}$ range covered, although this conclusion may be somewhat uncertain for $\frac{t}{a}=0.2$. The maximum tensile stress occurs in a location in a part of the seven-hole element which is quite similar to the single-hole hexagonal-element symmetry section.

$\underline{\text { Plate-Type Element }}$

In addition to the hexagonal elements, two plate configurations were investigated. These element shapes are shown in Figure 6. For these studies only the corner regions shown were included. The plate was terminated as shown and a symmetry condition was 


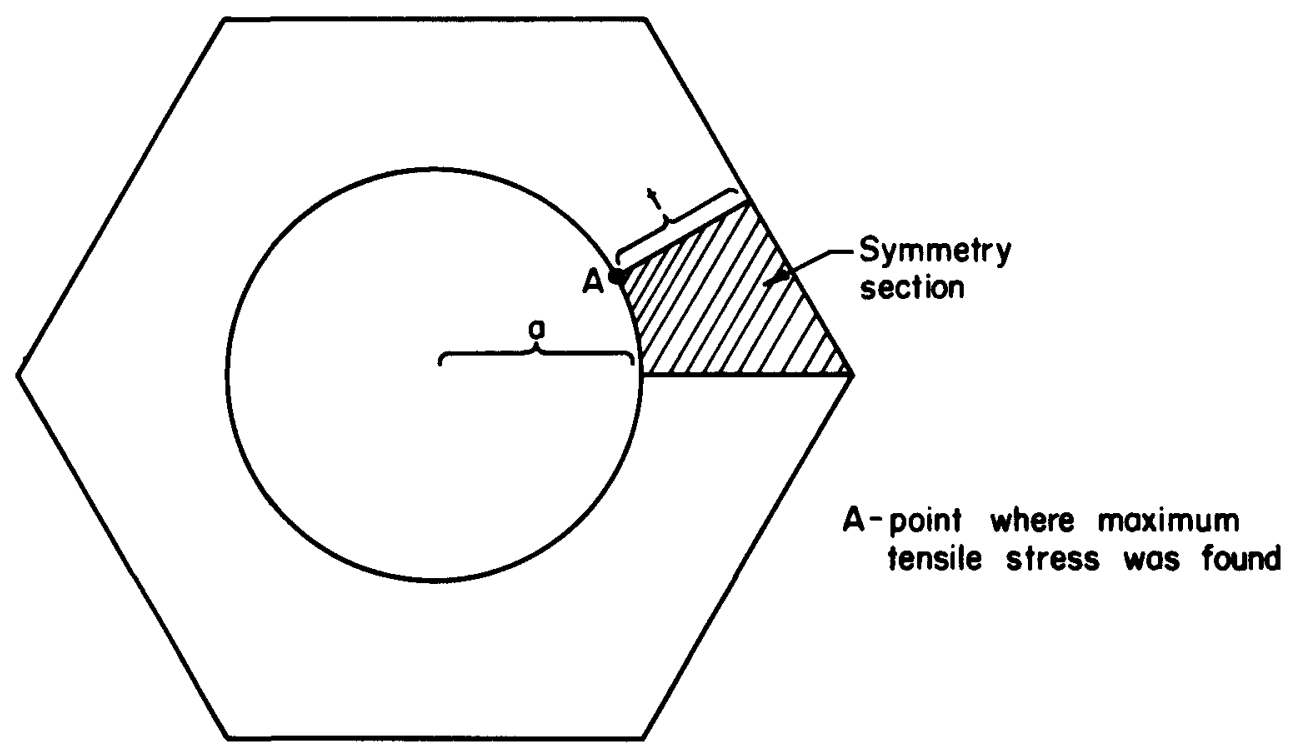

FIGURE 3. SINGLE-HOLE HEXAGONAL FUEL ELEMENT

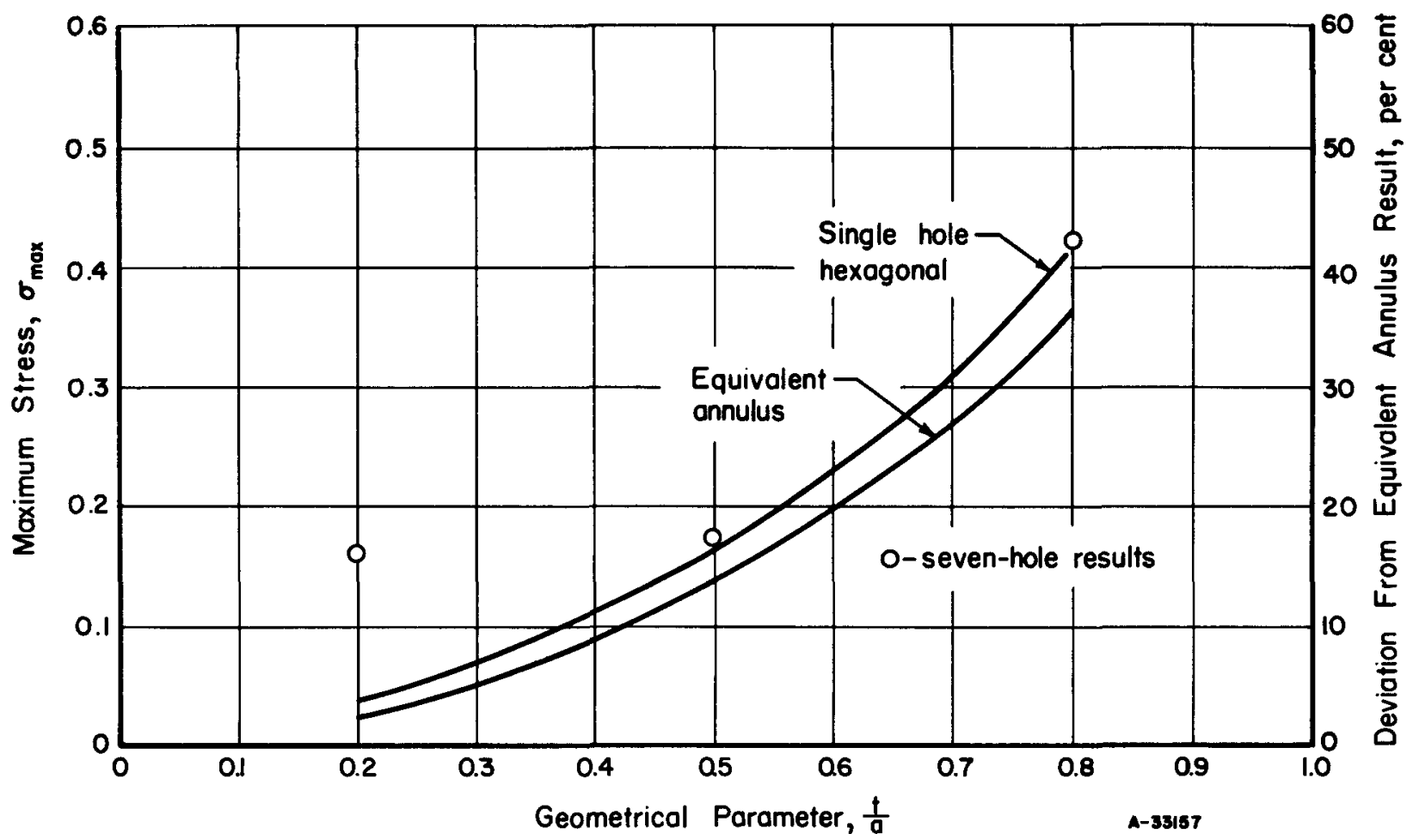

FIGURE 4. COMPARISON OF MAXIMUM STRESSES BETWEEN SINGLE-HOLE AND SEVEN-HOLE HEXAGONAL ELEMENTS AND THE EQUIVALENT ANNULUS 


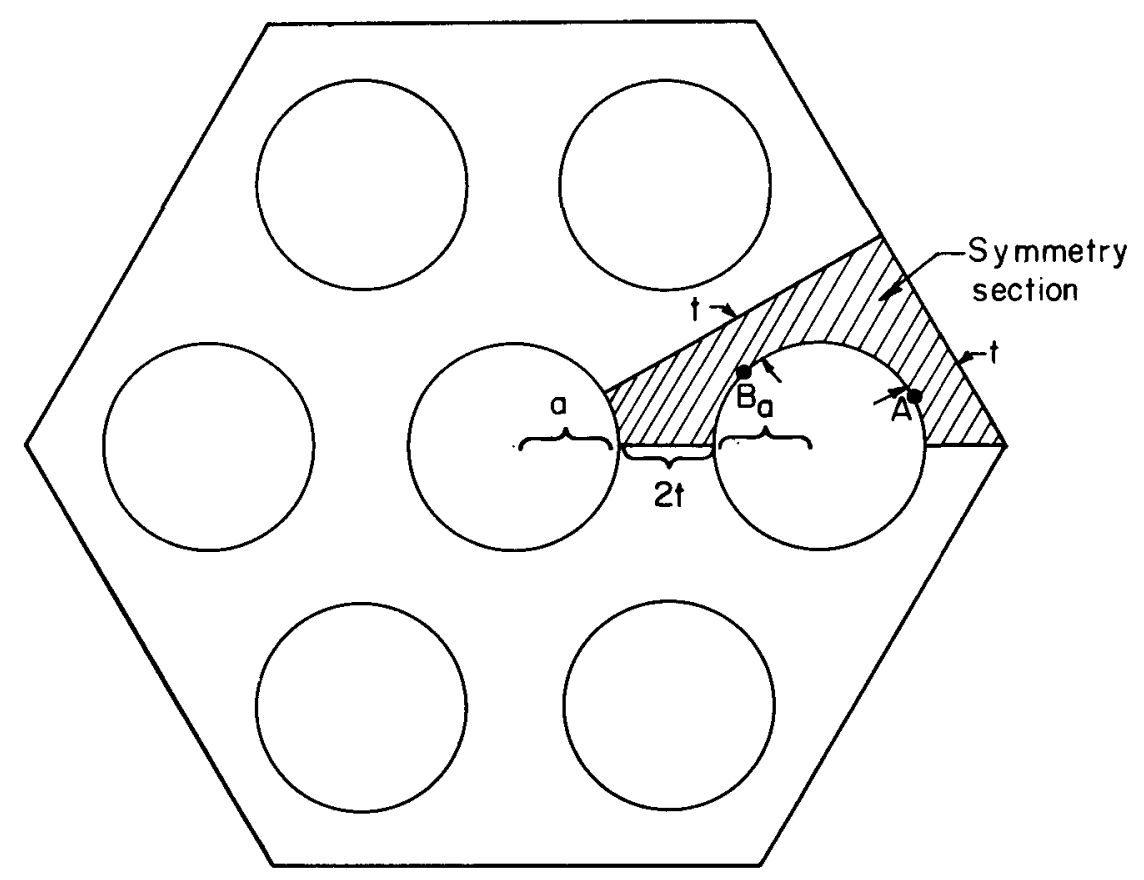

FIGURE 5. SEVEN-HOLE HEXAGONAL FUEL ELEMENT

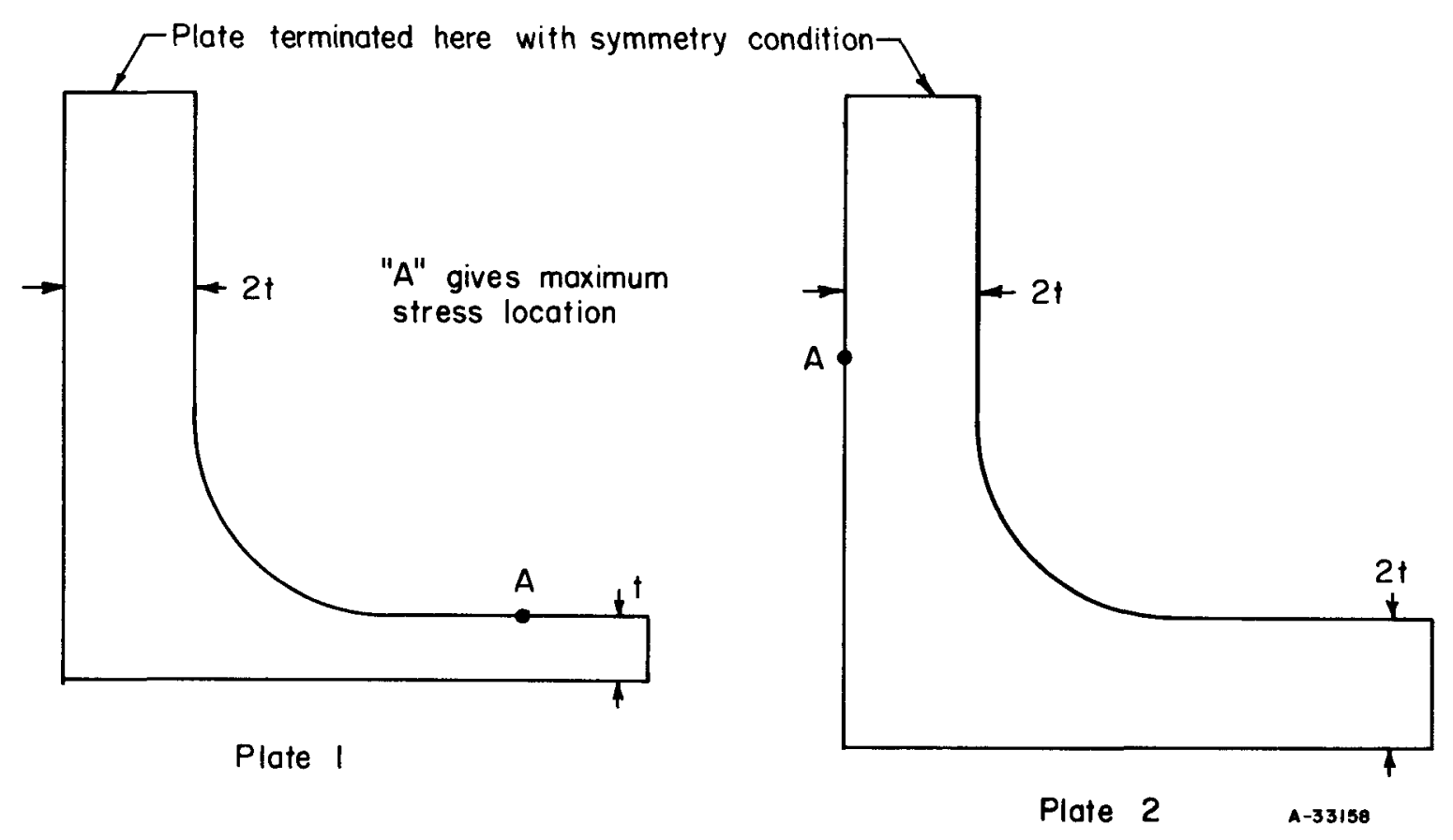

FIGURE 6. PLATE-TYPE FUEL ELEMENTS 
applied. All other surfaces were regarded as free surfaces. For these studies only the $\sigma_{x}, \sigma_{y}, \tau_{x y}$ stress components were of significance since the $\sigma_{z}$ distribution depends upon the average temperature over the entire plate. The maximum dimensionless stresses were found as given in Table 7 in the regions noted in Figure 6.

\section{TABLE 7. MAXIMUM TENSILE STRESS (a) IN PLATES}

\begin{tabular}{|c|c|c|}
\hline & $\frac{\sigma_{\max }}{\frac{\alpha E}{1-\nu} \frac{Q^{2}}{k}}$ & \\
\hline & Maximun & Tensile Stress, $\sigma$ \\
\hline Plate 1 & & 0.379 \\
\hline Plate 2 & & 0.350 \\
\hline
\end{tabular}

(a) These stresses are made dimensionless using for " $\mathrm{a}$ " the plate half thickness.

\section{DISCUSSION OF RESULTS AND CONCLUSIONS}

The numerical approach to the solution of thermal-stress problems as described in this report has been shown to be quite satisfactory in principle. The use of interpolation formulas to specify linear algebraic equations involving the unknown values of a function at mesh points has been described. The solution of the resulting set of equations has been shown to give rather good results even for a rather coarse mesh.

An iterative procedure has been described which gua rantees convergence and tends to smooth the function. However, the convergence of the method is such that rather close initial values must be determined if reasonable computer running times are used.

Computer codes have been developed which generate the required matrix elements, carry out the approximate iterative solutions, and process the solutions to yield the desired stresses.

The results obtained by applying these numerical procedures to a number of problems indicate that a more satisfactory method of solving the matrix equations is desirable. Several possibilities for making this improvement might be indicated here. One possibility is to employ a direct matrix-inversion method either for the complete problem or for a coarse-mesh problem. The method coded by Boehm(6) which employs matrix partitioning appears to be the most satisfactory for systems which may have up to 375 equations. Running-time estimates are not available. However, it was noted that a particular system of 158 equations required $30 \mathrm{~min}$ on the IBM-704. If running times with 375 equations are prohibitive or if more than 375 mesh points are considered, the matrix-inversion method could be applied to a coarser mesh. The solution from the coarse-mesh problem obtained in this way could then be used to interpolate a distribution for a finer mesh. This distribution could then be used as a good initial guess to 
use with the iteration routine described in this report. Even the coarse-mesh solution might be improved by iterating it with the iteration routine to reduce the round-off error incurred in the matrix-inversion process.

As indicated in the report previously, the optimum strategy might be to change the value of $\alpha$ during the iteration process. A more thorough study of the effect of different $\alpha$ choices might lead to a procedure with improved convergence.

Alternate iteration schemes might also be considered. However, the integral equations and boundary conditions as treated in this report make the usual line methods somewhat impractical. It is possible, however, to extend the minimization scheme of iteration to consider simultaneous changes of the values along a line or in a block. However, these extensions of the method also require further study before their possible worth can be determined. It can certainly be said though that the residual vector length can be reduced at least as much by a simultaneous change of $n$-values as it can by $n$ consecutive changes of the n-values. Of course, the advantage would be if the simultaneous change would lead to a much larger decrease in the length of the residual vector. Naturally, the gain achieved would have to have more than balance the increased complexity of the arithmetic.

While these suggested devices might prove advantageous, the most practical scheme seems to be the terminal block extrapolation method already described in this report. This method appears to offer high promise for a usable iteration scheme without the need for a close initial guess. Some small-size problems indicate that large gains can be made in accelerating the convergence by employing this method. Studies need to be made, however, with larger scale systems before the terminal block extrapolation can be evaluated.

\section{REFERENCES}

(1) Timoshenko, S., and Goodier, J. N., Theory of Elasticity, McGraw-Hill Book Company, Inc., 2nd Edition, New York (1951).

(2) Southwell, R. V., Relaxation Methods in Theoretical Physics, Clarendon Press, Oxford (1956).

(3) Hildebrand, F. B., Introduction to Numerical Analysis, McGraw-Hill Book Company, Inc., New York (1956).

(4) Wachspress, E. L., "Iterative Methods for Solving Elliptic-Type Differential Equations With Application to Two-Space-Dimension Multigroup Analysis", KAPL1333 (May I1, 1955).

(5) Keller, H. B., "On Some Iterative Methods for Solving Elliptic Difference Equations", AEC Computing Center, N. Y. U., NYO-7971 (September 1, 1957).

(6) Boehm, Barry, Share Program CA033 MEL $\phi$ (Matrix Equation, Large Order). 
•

. 
APPENDIX A

SETUP OF PROBLEM FOR MACHINE COMPUTATION 


\section{APPENDIX A}

\section{SETUP OF PROBLEM FOR MACHINE COMPUTATION}

It is the purpose of this appendix to present the manner of setup to be employed in order to prepare a physical problem for IBM-704 computation. As an illustration the complete specification is given of the annulus problem shown in Figure 1 in the text. It is not the intention here to supply running instructions for the machine programs.

\section{Problem Subroutines}

The normal problem involves the use of six programs which can be used separately or in series. These programs are presented below. The input terms are described later.

\section{Program 1. The Iteration Program}

This program requires as input the coefficient matrix and the transpose of the coefficient matrix on tape and the initial guess for the solution vector on punched cards. Also, on punched cards the number of iterations is specified, the $\alpha$-value is specified, and the printout frequency is specified. The input tape is prepared by Program 4 and the punched cards are prepared by hand. The output tape is used either in Program 5 or Program 6 or both.

Tape Input: Tape from Logical 6 of Program 4 should be mounted on Logical 6.

Tape Output: Tape from Logical 7

Printout: At every printout cycle the solution vector, the change in the solution vector, and the residual vector are listed. In addition, the square of the residual vector is listed.

Program 2. The Coefficient-Matrix Program

for the Temperature Problem

This program prepares the coefficient matrix from the input information regarding the geometry of the body and the mesh work.

Card Input:

Fixed Point - Fortran DIP

IE, IB, IC, NMAX, MMAX, IPMSN, ICN, IBDSN, IEXPNS, ITABCO, MA, NA, IBDSNA.

Floating Point - Fortran DIP

HCK, RA, SA, XNA, YNA. 
Output: On Logical Tape 3 will be written the matrix elements and their associated column indices. Also, any error-detection messages will be written on Logical Tape 3. On Logical Tape 6 will be written in binary the matrix elements and their associated column indices, one row per record. Logical Tape 6 is saved and used as input for Program 4.

Printout: The input data and the output data are listed.

Program 3. The Coefficient-Matrix Program

for the Stress Problem

This program prepares the coefficient matrix from the input information regarding the geometry of the body and the mesh work.

Card Input:

Fixed Point - Fortran DIP

MMAX, NMAX, IE, IB, IC, NI, N2, N3, N4, IPMSN, ICN, IBDSN, IEXPNS, ITABCO, MA, NA, IBDSNA.

Floating Point - Fortran DIP

RTSD 1, RTSD 2, RTSD 3, X $\phi U T, S C, R 1, X \phi, R A, S A$, XNA, YNA.

Output: On Logical Tape 3 will be written to matrix elements and their associated column indices. Also, any error-detection messages will be written on Logical Tape 3. On Logical Tape 6 will be written in binary the matrix elements and their associated column indices, one row per record. Logical Tape 6 is saved and used as input for Program 4.

Printout: The input data and the output data a re listed.

Program 4. Transpose-Matrix Program

This program prepares the transpose matrix and prepares a tape for the input to Program 1.

Tape Input: Tape from Logical 6 of Program 2 or 3 should be mounted on Logical 6 for this program.

Card Input:

Fixed Point - Fortran DIP

IE. 


\section{A-3}

Output: The original matrix, with column indices, and the transpose matrix, with column indices, are written on Logical Tape 3. The same information is written in binary on Logical Tape 5 which is saved and used as input to Program 1.

Printout: The matrix elements and the transpose-matrix elements are listed.

Program 5. Temperature-Data-

Processing Program

This program uses the temperature solution to generate the temperature terms of the integral equations: Equations (11), (13), and (14).

Card Input:

Fixed Point - Fortran DIP

N1, N2, N3, N4, NMAX, MMAX, IB, IC, IE, MA, NA, IBDSNA, ITABCO.

Floating Point - Fortran DIP

$\mathrm{X} \phi \mathrm{UT}, \mathrm{SC}, \mathrm{Rl}, \mathrm{HCK}, \mathrm{RA}, \mathrm{SA}, \mathrm{XNA}, \mathrm{YNA}$.

Tape Input: Tape from Logical 7 of Program 1 for the temperature solution should be mounted on Logical 6 for this program.

Output: The values for the temperature terms, called the right-hand sides, of the integral equations in Program 3 will be written on Logical Tape 3. They will be designated as RHS 1, RHS 2, and RHS 3. These values should be punched in Fortran DIP, FloatingPoint format and designated as RTSD 1, RTSD 2, and RTSD 3, respectively, and used as input in Program 3. Any errordetection messages will be written on Logical Tape 3. Logical Tape 6 should be saved and used as input to Program 6.

\section{Program 6. Data-Processing Program}

This program processes the temperature and stress-function solutions and determines the stress components at the interior mesh points.

Card Input:

Fixed Point - Fortran DIP

IE, MMAX, NMAX, IPMSN, ITABCO.

Floating Point - Fortran DIP

$\mathrm{V}, \mathrm{R} \phi$. 
Tape Input: The tape from Logical 7 of Program 1 for the stress problem should be mounted on Logical 6. The tape from Logical 7 of Program 1 for the temperature problem should be mounted on Logical 7 for this program.

Output: $\quad$ The average temperature, TBAR, for the body is written on Logical Tape 3. Also, the stress distribution and the temperature distribution for the body are written on Logical Tape 3. The maximum and minimum stresses are written on Logical Tape 9, which should be listed.

\section{Input Terminology}

Terms Only Applicable to Annular, Single-

Hole, and Seven-Hole Hexagonal Symmetry

Elements When Oriented as in Figures

N1 - Number of boundary points on center-hole boundary (including end points)

N2 - Number of boundary points on diagonal radial-symmetry line (including end points)

N3 - Number of boundary points on outer free boundary (including end points)

N4 - Number of boundary points on off-center hole boundary (including end points); $\mathrm{N} 4=0$ for single-hole problems

$\mathrm{R} \phi \quad-$ Radius of center hole in mesh units

R1 - Radius of off-center hole in mesh units

$\mathrm{X} \phi \quad-\mathrm{X}$-distance of left-most point of square mesh from center of the center hole in mesh units

XOUT - X-distance of right-most point of body from center of central hole in mesh units

SC - X-distance of off-center hole center from center of central hole in mesh units ( single-hole case $\mathrm{SC}=\mathrm{X} 0+2$ )

General Terms

MMAX - Total number of rows in rectangle of the square mesh

NMAX - Total number of columns in rectangle of the square mesh

IE - Total number of equations in the system = total number of the physical mesh points 
IB - Total number of physical mesh points for which harmonic or biharmonic equations are not written

IC - Total number of boundary points

$\mathrm{HCK} \quad-\frac{h \mathrm{c}}{\mathrm{k}}=\beta$

$\mathrm{V} \quad$ - Poisson's ratio

Correspondence Table

ITABCO - This table (IE entries) lists the sequence numbers of the geometrical mesh points in the sequence corresponding to the physical mesh points. The geometrical-mesh-point sequence starts at the upper left corner of the rectangle and continues from left to right row by row until the lower right corner of the rectangle is reached. The physical-mesh sequence starts at the physical mesh point with the smallest geometrical sequence number and proceeds by numbering the physical mesh points according to their order in the geometrical sequence. The physical mesh points are those used in the physical problem, i. e., interior and exterior points. The geometrical mesh includes all the mesh points in the covering rectangle (see Figure 1 in text).

\section{Geometry Tables}

IBDSNA - This table lists the boundary-point sequence numbers in sequence; hence, this table is the sequence $1,2,3, \ldots$, IC.

RA - This table (IC entries) lists the $\Delta x$ displacement of each boundary point from the selected interior expansion point in mesh units.

SA - This table (IC entries) lists the $\Delta y$ displacement of each boundary point from the selected interior expansion point in mesh units.

XNA - This table (IC entries) lists the $\mathrm{x}$-component of the unit outward normal vector at each boundary point.

YNA - This table (IC entries) lists the y-component of the unit outward normal vector at each boundary point.

MA - This table (IC entries) lists the row number of the interior expansion point for each boundary point.

NA - This table (IC entries) lists the column number of the interior expansion point for each boundary point. 
Equation Tables

IPMSN - This table (IB entries) lists in order the physical-mesh sequence numbers which are to be considered as exterior mesh points.

ICN - This table (IB entries) lists the number of the equation type to be written for each exterior point. The ordering in this table corresponds to the IPMSN table.

IBDSN - This table (IB entries) lists the boundary-point number at which the boundary equation will be written for each exterior point.

IEXPNS - This table (IB entries) lists the physical-mesh-point sequence number about which the boundary point is expanded for each exterior point.

Equation Type Numbers

The necessary equations are utilized in the programs according to an equationtype number. These numbers are as follows:

Temperature Problem

Equation

Type Number

1

2

3

4

$\begin{aligned} & \text { Equation } \\ & \nabla^{2} \mathrm{~T}=-1 \\ & \frac{\partial \mathrm{T}}{\partial \mathrm{n}}=0 \\ & \frac{\partial \mathrm{T}}{\partial \mathrm{n}}=-\beta \mathrm{T} \\ & \mathrm{T}=0\end{aligned}$

Stress Problem

Equation

Type Number

1

2

3

4

5

6
Equation

$$
\nabla^{4} \phi=1
$$

$$
\phi=0
$$$$
\frac{\partial \phi}{\partial \mathrm{n}}=0
$$$$
\frac{\partial^{2} \phi}{\partial n}=0
$$

$\frac{\partial \phi}{\partial y}=0$

$\frac{\partial \phi}{\partial x}=0$ 
Equation

Type Number

7

8

9

10

\section{Equation}

$$
\begin{gathered}
\frac{\partial \phi}{\partial \mathrm{x}}=\frac{\partial \phi}{\partial \mathrm{x}} \text { at specified point } \\
\oint \frac{\partial}{\partial \mathrm{n}}\left\{\nabla^{2} \phi+\mathrm{T}\right\} \mathrm{d} \mathbf{s}=0 \text { on center hole } \\
\oint \frac{\partial}{\partial \mathrm{n}}\left\{\nabla^{2} \phi+\mathrm{T}\right\} \mathrm{ds}=0 \text { on off-center hole } \\
\oint\left[\mathrm{x} \frac{\partial}{\partial \mathrm{n}}-\mathrm{n}_{\mathrm{x}}\right]\left[\nabla^{2} \phi+\mathrm{T}\right] \mathrm{d} \mathbf{s}=0 \text { on off-center hole }
\end{gathered}
$$

Example of Problem Setup for Simple Annulus

As an example of the setup for a typical problem, consider the simple annulus of Figure 1 in the text. The following data are immediately obtained:

$$
\begin{aligned}
\mathrm{N} 1 & =4 \\
\mathrm{~N} 2 & =4 \\
\mathrm{~N} 3 & =5 \\
\mathrm{~N} 4 & =0 \\
\mathrm{R} \phi & =10.0 \\
\mathrm{R} 1 & =0 \\
\mathrm{X} \phi & =8.0 \\
\mathrm{XOUT} & =14.0 \\
\mathrm{XC} & =10.0 \\
\mathrm{MMAX} & =8 \\
\text { NMAX } & =9 \\
\mathrm{IE} & =51 \\
\mathrm{IB} & =36 \\
\mathrm{IC} & =20
\end{aligned}
$$


A-8

Input Tables

\begin{tabular}{|c|c|c|c|c|c|}
\hline \multirow{2}{*}{$\begin{array}{c}\text { Sequence } \\
\text { Number }\end{array}$} & \multirow[b]{2}{*}{ IPMSN } & \multicolumn{2}{|c|}{ ICN } & \multirow[b]{2}{*}{ IBDSN } & \multirow[b]{2}{*}{ IEXPNS } \\
\hline & & Temperature & Stress & & \\
\hline 1 & 1 & 4 & 4 & 7 & 11 \\
\hline 2 & 2 & 4 & 4 & 8 & 12 \\
\hline 3 & 3 & 4 & 4 & 5 & 17 \\
\hline 4 & 4 & 4 & 4 & 6 & 18 \\
\hline 5 & 5 & 2 & 3 & 7 & 11 \\
\hline 6 & 6 & 2 & 3 & 8 & 12 \\
\hline 7 & 7 & 4 & 6 & 9 & 12 \\
\hline 8 & 8 & 4 & 6 & 4 & 17 \\
\hline 9 & 9 & 2 & 3 & 5 & 17 \\
\hline 10 & 10 & 2 & 3 & 6 & 18 \\
\hline 11 & 13 & 2 & 5 & 10 & 12 \\
\hline 12 & 14 & 4 & 6 & 10 & 12 \\
\hline 13 & 15 & 4 & 6 & 3 & 17 \\
\hline 14 & 16 & 3 & 5 & 3 & 17 \\
\hline 15 & 21 & 2 & 5 & 11 & 20 \\
\hline 16 & 22 & 4 & 6 & 11 & 20 \\
\hline 17 & 23 & 4 & 6 & 2 & 25 \\
\hline 18 & 24 & 3 & 5 & 2 & 25 \\
\hline 19 & 29 & 2 & 5 & 12 & 28 \\
\hline 20 & 30 & 4 & 6 & 12 & 28 \\
\hline 21 & 31 & 4 & 6 & 1 & 33 \\
\hline 22 & 32 & 3 & 8 & 1 & 33 \\
\hline 23 & 38 & 2 & 6 & 13 & 37 \\
\hline 24 & 39 & 4 & 2 & 13 & 37 \\
\hline 25 & 40 & 4 & 5 & 20 & 33 \\
\hline 26 & 41 & 2 & 3 & 19 & 33 \\
\hline 27 & 42 & 2 & 3 & 18 & 34 \\
\hline 28 & 43 & 2 & 3 & 17 & 35 \\
\hline 29 & 44 & 2 & 3 & 16 & 36 \\
\hline 30 & 45 & 2 & 3 & 15 & 37 \\
\hline 31 & 46 & 4 & 5 & 14 & 37 \\
\hline 32 & 47 & 4 & 4 & 19 & 33 \\
\hline 33 & 48 & 4 & 4 & 18 & 34 \\
\hline 34 & 49 & 4 & 4 & 17 & 35 \\
\hline 35 & 50 & 4 & 4 & 16 & 36 \\
\hline 36 & 51 & 4 & 4 & 15 & 37 \\
\hline
\end{tabular}

Physical-Mesh

Physical-Mesh

Sequence Number

ITABCO

Sequence Number

$\begin{array}{ll}11 & 23 \\ 12 & 24 \\ 13 & 25 \\ 14 & 26 \\ 15 & 28 \\ 16 & 29 \\ 17 & 30 \\ 18 & 31 \\ 19 & 32 \\ 20 & 33\end{array}$




\begin{tabular}{cccc}
$\begin{array}{c}\text { Physical-Mesh } \\
\text { Sequence Number }\end{array}$ & ITABCO & $\begin{array}{c}\text { Physical-Mesh } \\
\text { Sequence Number }\end{array}$ & ITABCO \\
\cline { 1 - 2 } 21 & 34 & 37 & 52 \\
22 & 35 & 38 & 53 \\
23 & 37 & 49 & 54 \\
24 & 38 & 41 & 56 \\
25 & 39 & 42 & 57 \\
26 & 40 & 43 & 58 \\
27 & 41 & 44 & 59 \\
28 & 42 & 45 & 60 \\
29 & 43 & 46 & 61 \\
30 & 44 & 47 & 62 \\
31 & 46 & 48 & 66 \\
32 & 47 & 49 & 67 \\
33 & 48 & 50 & 68 \\
34 & 49 & 51 & 69 \\
35 & 50 & & 70 \\
36 & 51 & &
\end{tabular}

Discussion of Example Problem Setup

It will be apparent that the problem specification given is not unique. However, this nonuniqueness should present no problems as long as independent equations are utilized. Several points may be elaborated, however.

Boundary Points 14 and 20 are actually not on the boundary. They are specified for convenience in order to write for physical Mesh Points 46 and 40 a symmetry condition in the form of equation Type 5 for the stress problem.

The association of IPMSN 32 with IBDSN 1 and IEXPNS 33 for the stress problem is somewhat ambiguous since ICN 8 is the integral equation. However, the association is meaningful for the temperature problem and the tables are kept identical in the temperature and stress problems where possible. Actually, for the integral equations the information given in IBDSN and IEXPNS is not used.

It should also be noted that entirely different tables of ICN must be used for the temperature and stress problems. The other tables may be different or may be identical. However, the ITABCO tables must be identical in the two problems. This, of course, means that trivial points are introduced in the temperature problem which are eliminated by temperature equation Type 4 at these points.

The equation Type 5 and 6 in the stress problem are Equations (9c) which can replace Equations (9b). This follows since by Equations (9c)

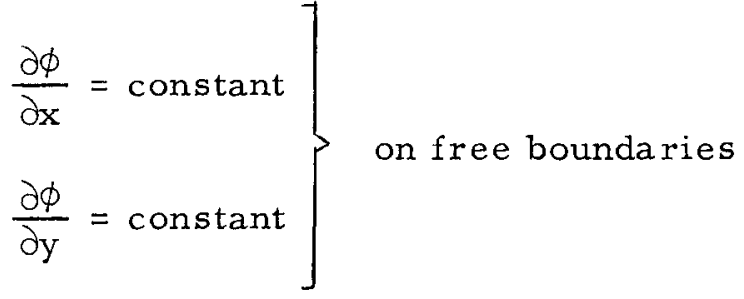




\section{A- 10}

These constants must be zero for both free boundaries since at the ends of both free boundaries a symmetry surface is met where $\frac{\partial \phi}{\partial n}=0$ for a different normal direction. In the case of the seven-hole hexagonal element only $\frac{\partial \phi}{\partial y}=0$ for the off-center hole boundary and, hence, equation Type 7 is also needed here, i. e., $\frac{\partial \phi}{\partial x}=\frac{\partial \phi}{\partial x}$ at specified point on the boundary or $\frac{\partial \phi}{\partial x}=$ constant. 